\title{
An ALMA Multiline Survey of the Interstellar Medium of the Redshift 7.5 Quasar Host Galaxy J1342+0928
}

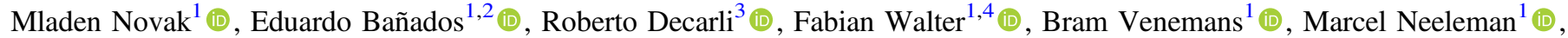

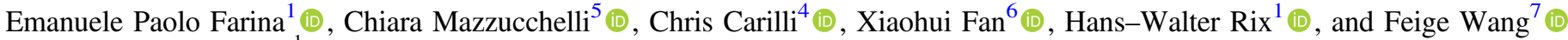 \\ ${ }^{1}$ Max-Planck-Institut für Astronomie, Königstuhl 17, D-69117 Heidelberg, Germany; novak@mpia.de \\ ${ }^{2}$ The Observatories of the Carnegie Institution for Science, 813 Santa Barbara Street, Pasadena, CA 91101, USA \\ ${ }_{3}^{3}$ INAFOsservatorio di Astrofisica e Scienza dello Spazio, via Gobetti 93/3, I-40129, Bologna, Italy \\ ${ }^{4}$ National Radio Astronomy Observatory, Pete V. Domenici Array Science Center, P.O. Box O, Socorro, NM 87801, USA \\ ${ }^{5}$ European Southern Observatory, Alonso de Córdova 3107, Vitacura, Región Metropolitana, Chile \\ ${ }^{6}$ Steward Observatory, The University of Arizona, 933 N. Cherry Ave., Tucson, AZ 85721, USA \\ ${ }^{7}$ Department of Physics, University of California, Santa Barbara, CA 93106-9530, USA \\ Received 2019 April 12; revised 2019 June 18; accepted 2019 June 20; published 2019 August 13
}

\begin{abstract}
We use Atacama Large Millimeter Array observations of the host galaxy of the quasar ULAS J1342+0928 at $z=7.54$, to study the dust continuum and far-infrared lines emitted from its interstellar medium (ISM). The Rayleigh-Jeans tail of the dust continuum is well sampled with eight different spectral setups, and from a modified blackbody fit we obtain an emissivity coefficient of $\beta=1.85 \pm 0.3$. Assuming a standard dust temperature of $47 \mathrm{~K}$ we derive a dust mass of $M_{\text {dust }}=0.35 \times 10^{8} M_{\odot}$ and a star formation rate of $150 \pm 30 M_{\odot} \mathrm{yr}^{-1}$. We have $>4 \sigma$ detections of the $[\mathrm{C} \mathrm{II}]_{158 \mu \mathrm{m}}$, $[\mathrm{O} \mathrm{III}]_{88 \mu \mathrm{m}}$, and $[\mathrm{N} \mathrm{II}]_{205 \mu \mathrm{m}}$ atomic fine structure lines and limits on the $[\mathrm{C} \mathrm{I}]_{369 \mu \mathrm{m}},[\mathrm{O} \mathrm{I}]_{146 \mu \mathrm{m}}$, and [N II $]_{205} \mu \mathrm{m}$ emission. We also report multiple limits of CO rotational lines with $J_{\text {up }} \geqslant 7$, as well as a tentative $3.3 \sigma$ detection of the stack of four CO lines $\left(J_{\text {up }}=11,10,8\right.$, and 7$)$. We find line deficits that are in agreement with local ultra-luminous infrared galaxies. Comparison of the $[\mathrm{N} \mathrm{II}]_{205} \mu \mathrm{m}$ and $[\mathrm{C} \mathrm{III}]_{158 \mu \mathrm{m}}$ lines indicates that the $[\mathrm{C} \mathrm{II}]_{158 \mu \mathrm{m}}$ emission arises predominantly from the neutral medium, and we estimate that the photodisassociation regions in J1342+0928 have densities $\lesssim 5 \times 10^{4} \mathrm{~cm}^{-3}$. The data suggest that $\sim 16 \%$ of hydrogen is in ionized form and that the H II regions have high electron densities of $n_{e}>180 \mathrm{~cm}^{-3}$. Our observations favor a low gas-to-dust ratio of $<100$, and a metallicity of the ISM comparable to the solar value. All the measurements presented here suggest that the host galaxy of J1342+0928 is highly enriched in metal and dust, despite being observed just $680 \mathrm{Myr}$ after the big bang.
\end{abstract}

Key words: cosmology: observations - galaxies: high-redshift - galaxies: individual (ULAS J1342+0928) galaxies: ISM - quasars: emission lines

\section{Introduction}

Observations of the early universe present a critical piece of information for the overall picture of galaxy evolution. Technical advancements continually push the observational boundaries and fainter, more distant, objects become detectable with a reasonable investment of telescope time. Quasars, being the most luminous nontransient shining light sources, present natural targets for early universe investigations. The large energy output of the quasar arises from rapid accretion of material $\left(\gtrsim 10 M_{\odot} \mathrm{yr}^{-1}\right)$ onto a supermassive black hole (SMBH; mass greater than $\gtrsim 10^{8} M_{\odot}$ ) centered in the galaxy host (e.g., De Rosa et al. 2014).

Redshifts higher than $z \gtrsim 6$ correspond to the first gigayear of the universe, which is of particular interest as it overlaps with the last phase change of the universe: the reionization epoch (see, e.g., Becker et al. 2015). Several hundred quasars were found at these cosmic times, owing to large survey programs (e.g., Fan et al. 2006; Bañados et al. 2016; Jiang et al. 2016; Matsuoka et al. 2018, and references therein). These observations of high-redshift quasars constrain the black hole seed masses and their growth in the early universe (e.g., Volonteri 2012), but also challenge the models of galaxy mass buildup (see also Sijacki et al. 2015; van der Vlugt \& Costa 2019).

A study of atomic fine structure emission lines can provide a plethora of information on the physical properties of the interstellar medium (ISM; see Carilli \& Walter 2013 for a review). For high-redshift galaxies, several far-infrared (FIR) emission lines of the most abundant atom/ion species, carbon $(\mathrm{C})$, oxygen $(\mathrm{O})$, and nitrogen $(\mathrm{N})$, are conveniently shifted into the millimeter and submillimeter atmospheric windows accessible to facilities such as the NOrthern Extended Millimeter Array (NOEMA) and the Atacama Large Millimeter Array (ALMA). The singly ionized carbon line, $[\mathrm{C} \mathrm{II}]_{158 \mu \mathrm{m}}$, the brightest FIR emission line emitted in both neutral and ionized medium, is an important coolant of the ISM and a good tracer of gas kinematics. These properties led to systematic targeting of the $[\mathrm{C} \mathrm{II}]_{158 \mu \mathrm{m}}$ resulting in detections of dozens of $z>6$ quasars (e.g., Wang et al. 2013; Willott et al. 2015; Venemans et al. 2017a; Decarli et al. 2018). Although [C II $]_{158 \mu \mathrm{m}}$ remains the main diagnostic line of the ISM for high-redshift quasars, other fine-structure lines can be observed to provide additional constraints on the physical properties of the ISM in these sources.

Recently, several studies were aimed at observing doubly ionized oxygen high-redshift galaxies and quasars, specifically the $[\mathrm{O} \mathrm{III}]_{88 \mu \mathrm{m}}$ line (e.g., Carniani et al. 2017; Hashimoto et al. 2018; Marrone et al. 2018; Walter et al. 2018). Given the high ionization energy required to produce $\mathrm{O}^{++}$emission, it originates exclusively in an ionized medium around early-type stars or in the presence of active galactic nuclei (AGNs). This line shows a promising future for high-redshift galaxy 
Table 1

Frequency Setups of Our ALMA Observations of J1342+0928 and Properties of the Derived Continuum Maps, which Exclude Emission Line Channels

\begin{tabular}{|c|c|c|c|c|c|c|c|}
\hline Band & $\begin{array}{c}\nu_{\mathrm{obs}}^{\mathrm{a}} \\
(\mathrm{GHz})\end{array}$ & $\begin{array}{c}\text { Beam } \\
\left(\operatorname{arcsec}^{2}\right)\end{array}$ & $\begin{array}{c}\mathrm{rms} \\
\left(\mu \mathrm{Jy} \text { beam }^{-1}\right)\end{array}$ & $\begin{array}{c}\text { Aperture } S_{\nu} \\
(\mu \mathrm{Jy})\end{array}$ & Covered Emission Lines & $\begin{array}{l}\text { Observation } \\
\text { Date(s) }\end{array}$ & $\begin{array}{c}\text { Time on } \\
\text { source }\end{array}$ \\
\hline 3 & 101.3 & $0.92 \times 0.72$ & 7.5 & $<22.4^{\mathrm{b}}$ & $\mathrm{CO}(7-6),[\mathrm{C} \mathrm{I}]_{369 \mu \mathrm{m}}, \mathrm{CO}(8-7), \mathrm{OH}^{+}$ & 2018 Jan 16 & $67 \mathrm{~min}$ \\
\hline 5 (a) & 176.0 & $1.69 \times 1.07$ & 19 & $137 \pm 31$ & {$[\mathrm{~N} \mathrm{III}]_{205 \mu \mathrm{m}}$} & 2018 Aug $23 / 24$ & $71 \mathrm{~min}$ \\
\hline 5 (b) & 195.3 & $1.90 \times 1.33$ & 26 & $214 \pm 36$ & $\mathrm{CO}(14-13), \mathrm{CO}(15-14), \mathrm{H}_{2} \mathrm{O}$ & $2018 \mathrm{Jul} 4$ & $46 \mathrm{~min}$ \\
\hline 6 (a) & 221.8 & $1.11 \times 0.88$ & 19 & $257 \pm 42$ & $\mathrm{CO}(16-15), \mathrm{CO}(17-16), \mathrm{OH}, \mathrm{OH}^{+}$ & 2018 Apr 12 & $39 \mathrm{~min}$ \\
\hline 8 & 403.9 & $0.98 \times 0.59$ & 110 & $993 \pm 320$ & {$[\mathrm{O} \mathrm{IIII}]_{88 \mu \mathrm{m}}$} & 2018 May 12 & $22 \mathrm{~min}$ \\
\hline FIRST & 1.4 & $5.4 \times 5.4$ & 144 & $<432$ & & & \\
\hline VLA & 41 & $2.2 \times 2.0$ & 5.7 & $<17.1$ & & & \\
\hline
\end{tabular}

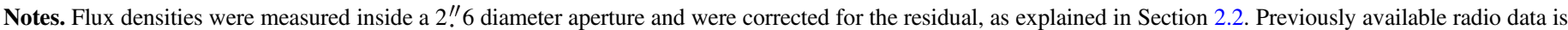
listed at the end for completeness (see Venemans et al. 2017b).

${ }^{a}$ Center of the entire frequency setup.

${ }^{\mathrm{b}}$ Upper limits correspond to $3 \sigma$ of the local rms noise.

observations (see also Hashimoto et al. 2019). Singly ionized nitrogen gives rise to two emission lines $[\mathrm{N} \mathrm{II}]_{205} \mu \mathrm{m}$ and $[\mathrm{N} \mathrm{III}]_{205 \mu \mathrm{m}}$, which can provide additional ionization diagnostics (see, e.g., Herrera-Camus et al. 2016), especially when used in conjunction with other lines. Observations of multiple fine structure lines at high redshifts are still rare (e.g., Tadaki et al. 2019).

Beside the abovementioned fine structure lines, useful ISM diagnostic lines can also originate in molecules (see, e.g., Carilli \& Walter 2013). The most abundant molecule, $\mathrm{H}_{2}$, is not practically observable and we rely on tracers such as carbon monoxide $\left({ }^{12} \mathrm{CO}\right)$ to measure the gas content of galaxies (for a review, see Bolatto et al. 2013). When multiple CO rotational line detections are available, it is possible to put constraints on the kinetic temperature of the gas (see, e.g., Daddi et al. 2015). Several studies of CO excitation ladders for high-redshift quasars were performed showing its usefulness in analyzing molecular gas excitation (e.g., Weiß et al. 2007; Riechers et al. 2009; Gallerani et al. 2014; Carniani et al. 2019). In denser regions, where it is more difficult to observe common gas tracers due to optical depth effects, the water $\left(\mathrm{H}_{2} \mathrm{O}\right)$ and the hydroxyl $\left(\mathrm{OH}, \mathrm{OH}^{+}\right)$molecules can provide valuable insight into the state of the ISM. Their emission probes heated regions of star formation or in the presence of an AGN (e.g., Liu et al. 2017), but it can also be tied to shocked regions and molecular outflows (e.g., Fischer et al. 2010; González-Alfonso et al. 2013). Due to the complex energy level diagram of water, multiple lines are necessary for a valid interpretation (see, e.g., van der Werf et al. 2011; Riechers et al. 2013).

In this work, we present a multiline search survey targeting the quasar host galaxy ULAS J1342+0928 (hereafter J1342 +0928), which to date holds the record as the most distant quasar observed. It was discovered by Bañados et al. (2018), who reported an absolute $\mathrm{AB}$ magnitude at $1450 \AA$ of $M_{1450}=-26.8$, bolometric luminosity of $L_{\mathrm{bol}}=10^{13} L_{\odot}$, and an SMBH mass of $8 \times 10^{8} M_{\odot}$. It was followed-up with NOEMA by Venemans et al. (2017b) in order to constrain the dust continuum and the ionized carbon emission. These observations resulted in the detection of bright $[\mathrm{C} \mathrm{II}]_{158 \mu \mathrm{m}}$ emission, and upper limits of several $\mathrm{CO}$ emission lines. For the $[\mathrm{C} \mathrm{III}]_{158 \mu \mathrm{m}}$ line, a redshift of $z=7.5413$, and a line width of $380 \mathrm{~km} \mathrm{~s}^{-1}$ were reported, which we adopt throughout. We here present ALMA observations targeting various emission lines of carbon, oxygen and nitrogen species, as well as molecular lines of carbon monoxide, hydroxyl and water in order to explore, in detail, the conditions of the ISM in the most distant quasar host galaxy known.

Throughout the paper we assume the concordance lambda cold dark matter $(\Lambda \mathrm{CDM})$ cosmology with the Hubble constant of $H_{0}=70 \mathrm{~km} \mathrm{~s}^{-1} \mathrm{Mpc}^{-1}$, dark energy density of $\Omega_{\Lambda}=0.7$, and matter density of $\Omega_{\mathrm{m}}=0.3$. At the redshift of the source $(z=7.5413)$ the age of the universe is $0.68 \mathrm{Gyr}$, and an angular size of $1^{\prime \prime}$ corresponds to $5.0 \mathrm{kpc}$.

\section{Data}

\subsection{Observations and Data Reduction}

We have observed the quasar $\mathrm{J} 1342+0928$ at $z=7.5413$ with ALMA using between 41 and 46 antennas that are $12 \mathrm{~m}$ in diameter, eight different frequency setups reaching an effective bandwidth of $60 \mathrm{GHz}$, located between $93.5 \mathrm{GHz}$ (band 3) and $412 \mathrm{GHz}$ (band 8), to cover 24 molecular and fine-structure emission lines (program ID: 2017.1.00396.S, PI: Bañados). Table 1 summarizes all observational setups along with the total (science) time spent on the source.

The default calibration pipeline was used to process the raw data with the Common Astronomy Software Applications (CASA) package (McMullin et al. 2007). Visibility data of various execution blocks were merged together and imaged in CASA using TCLEAN. We used natural weighting to maximize sensitivity in the resulting maps. Several imaging products were created, as follows. For each setup we produced a cube with a channel width corresponding to $50 \mathrm{~km} \mathrm{~s}^{-1}$ at the central frequency of the setup. We then subtracted the continuum estimated from all channels excluding the ones where emission lines might be detected using the UVCONTSUB task (covered emission lines are listed in Table 1).

Only the $[\mathrm{C} \mathrm{III}]_{158 \mu \mathrm{m}}$ observations have enough signal-tonoise $(\mathrm{S} / \mathrm{N})$ to model the shape of the line, specifically its width and precise redshift. These measurements were already performed with the low resolution NOEMA observations by Venemans et al. (2017b). For other lines we assume that the 
full width at half maximum (FWHM) remains the same (further discussed in Section 3.2). To maximize the measured signal of the other lines, we imaged integrated emission line maps (also known as moment zero maps) over a velocity width of $455 \mathrm{~km} \mathrm{~s}^{-1}$, corresponding to 1.2 times the FWHM of the $[\mathrm{C} \mathrm{II}]_{158 \mu \mathrm{m}}$ line, centered at the targeted line frequency redshifted to $z=7.5413$. A Gaussian fit to our $[\mathrm{C} \mathrm{III}]_{158 \mu \mathrm{m}}$ spectrum measured inside an aperture (as explained in the following section) across $50 \mathrm{~km} \mathrm{~s}^{-1}$ wide channels would result in a value of $z=7.5400 \pm 0.0005$, with line flux and width that are also in agreement with already published values. For consistency reasons, we adopt the redshift and the line width values reported in Venemans et al. (2017b) and use them throughout.

Additional $455 \mathrm{~km} \mathrm{~s}^{-1}$ wide channels were imaged on both sides of the moment zero line map in order to visually check that the continuum subtraction performed as expected. We also image the continuum part of each spectral setup (i.e., excluding line emission). Every map cleaning was performed inside a circle of $4^{\prime \prime}$ diameter located at the phase center, and another circle positioned on the foreground source in the field $10^{\prime \prime}$ away (in the NE direction), down to $2 \sigma$ of the rms noise inside the cube. The foreground source was cleaned to prevent its sidelobes from biasing our flux measurements, as it is detected in every continuum band that we have observed. We also detect several emission lines in this source, and their observed frequencies imply that the source is not related to J1342+0928. Further analysis of the foreground source is outside the scope of this paper.

\subsection{Measuring Flux Densities}

To ensure that we are probing the same spatial scales independent of the resolution of the maps, we extract flux densities for both the continuum and line emissions inside an aperture with a diameter of 2 !" 6 , corresponding to $13 \mathrm{kpc}$ at the redshift of the source. This approach yields measurements that describe average properties of the galaxy, which enables comparison in a consistent way. This aperture size was chosen in such a way to encompass all recoverable $[\mathrm{C} \mathrm{III}]_{158 \mu \mathrm{m}}$ extended emission, which was detected at the highest $\mathrm{S} / \mathrm{N}$ ratio and also at the highest resolution (see Table 1 and Appendix A). No significant emission was recovered for the continuum or other emission lines beyond the chosen aperture. We employ the residual scaling method when computing aperture flux densities in order to mitigate the issue of ill-defined units in interferometric maps and effects of sidelobes. We refer the reader to Appendix A for further technical details. Error estimate of the measured flux density is calculated as $\sigma \sqrt{N}$, where $\sigma$ is the local rms (in units of $\mathrm{Jy} \mathrm{beam}^{-1}$ ) and $N$ is the number of independent clean beams that fill the aperture.

\section{Results and Discussion}

\subsection{The Dust Continuum Emission}

The continuum emission of $\mathrm{J} 1342+0928$ was detected in seven out of eight observed spectral setups with a peak surface brightness $\mathrm{S} / \mathrm{N}>4$, while only an upper limit is available in the lowest frequency band. Continuum maps are shown in Figure 1, while flux density measurements and beam sizes are summarized in Table 1. Continuum emission is spatially coincident in all observed bands, and we define its center at the peak of the high-resolution $231.5 \mathrm{GHz}$ observations: $13^{\mathrm{h}} 42^{\mathrm{m}} 8^{\mathrm{s}} .098+9^{\circ} 28^{\prime} 38^{\prime \prime} 35$ (International Celestial Reference

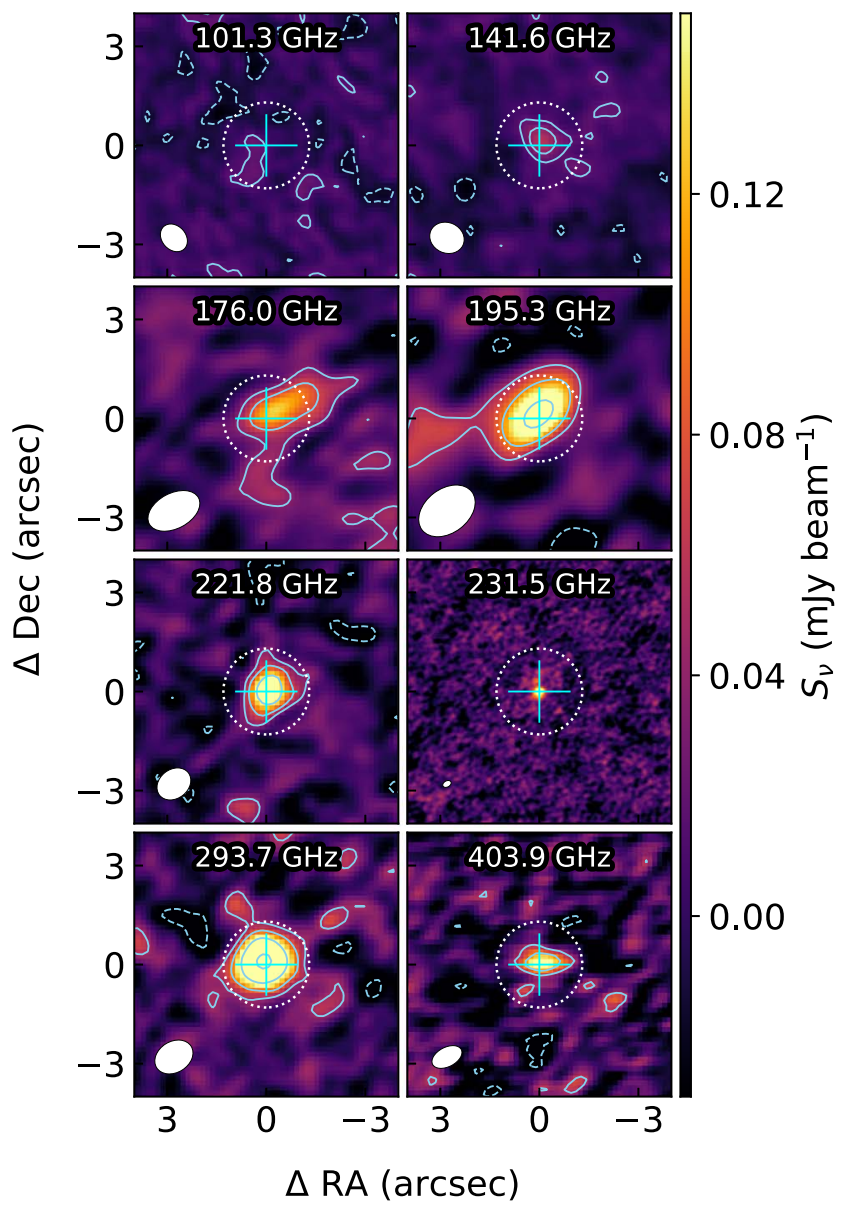

Figure 1. Continuum maps of eight different spectral setups of $\mathrm{J} 1342+0928$ (frequencies are given at the top of each panel). The respective synthesized beam sizes are shown in the lower left corner of each panel. The colorbar is multiplied by a factor of 0.5 (4) inside the 231.5 (403.9) $\mathrm{GHz}$ panel to facilitate better contrast. The white dotted circle represents the 2 ". 6 diameter aperture used for flux extraction. Full (dashed) contours outline $+(-) 2 \sigma, 4 \sigma, 8 \sigma$, and $16 \sigma$, where $\sigma$ is the rms noise (see Table 1). The cross marks the dust continuum center obtained from the high-resolution data $\left(13^{\mathrm{h}} 42^{\mathrm{m}} 8.098+9^{\mathrm{s}} 28^{\prime}\right.$ 38 "' 35 , ICRS).

System), also shown with a cross in Figure 1. The peak of the continuum coincides with the center measured in the $J$-band image obtained with the Magellan Baade telescope and corrected to Gaia DR2 astrometry, within the uncertainty of 50 mas. Two setups in band $5(176$ and $195.3 \mathrm{GHz})$ were observed at a lower resolution and appear slightly extended at $2 \sigma$ significance. We attribute this behavior to noise fluctuations, and note that our nominal aperture integral is still consistent within the error bars with a larger aperture measurement that would encompass more of this extended flux. We show the measured J1342+0928 spectral energy distribution (SED) in Figure 2 along with $3 \sigma$ upper limits on the radio continuum from the Faint Images of the Radio Sky at Twenty-cm (FIRST) survey and a targeted Karl G. Jansky Very Large Array observation (see Venemans et al. 2017b).

To constrain the FIR properties of $\mathrm{J} 1342+0928$, we fit a modified blackbody to our observations under the assumption that the dust optical depth is small at FIR wavelengths (optically thin regime). In this case the observed flux density originating from the heated dust can be expressed as

$$
S_{\nu_{\mathrm{obs}}}=f_{\mathrm{cmb}}(1+z) D_{L}^{-2} \kappa_{\nu_{\mathrm{rest}}} M_{\mathrm{dust}} B_{\nu_{\mathrm{rest}}}\left(T_{\mathrm{dust}, z}\right) \text {, }
$$




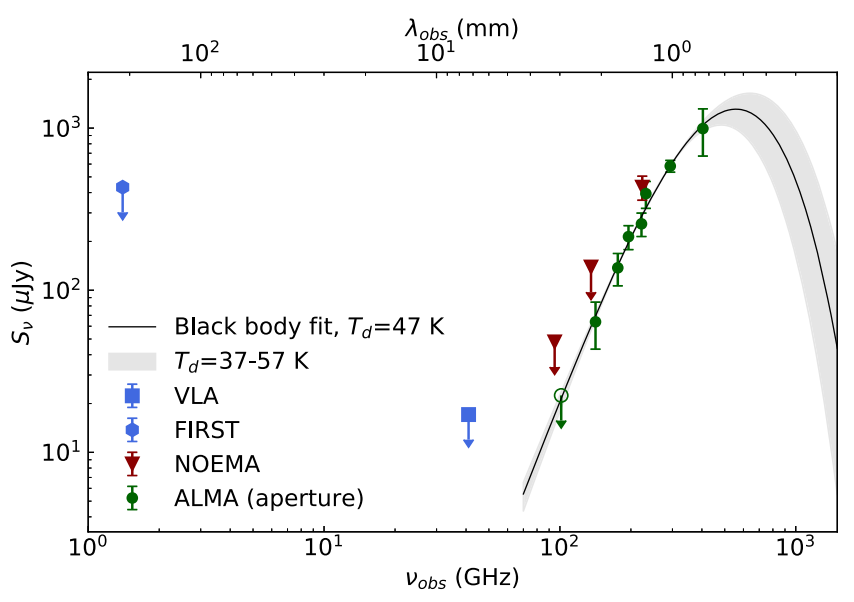

Figure 2. Spectral energy distribution of the dust continuum emission of J1342 +0928 . Our ALMA measurements are shown with green points. Only $3 \sigma$ upper limits are available for the radio continuum (Venemans et al. 2017b). A modified blackbody fit with intrinsic dust temperature of $47 \mathrm{~K}$ is shown with a black line, while the gray shaded area shows the fit for a wider range of temperatures (see the text for details).

where $f_{\mathrm{cmb}}$ is a measure of contrast against the cosmic microwave background (CMB) radiation, $D_{L}$ is the luminosity distance, $\kappa_{\nu_{\text {rest }}}$ is the dust mass opacity coefficient, $M_{\text {dust }}$ is the dust mass, $B_{\nu_{\text {rest }}}$ is the blackbody radiation spectrum, $T_{\text {dust }, z}$ is the temperature of the dust, which includes heating by the $\mathrm{CMB}$ at redshift $z$, and rest and observed frequencies are related via $\nu_{\text {rest }}=(1+\mathrm{z}) \nu_{\mathrm{obs}}$, with all values given in SI units. The opacity coefficient is defined as $\kappa_{0}$ (units of $\mathrm{m}^{2} \mathrm{~kg}^{-1}$ ) at a reference frequency $\nu_{0}$ with a power-law dependence on frequency

$$
\kappa_{\nu_{\text {rest }}}=\kappa_{\nu_{0}}\left(\nu_{\text {rest }} / \nu_{0}\right)^{\beta},
$$

where $\beta$ is the dust spectral emissivity index. Given the high redshift of $\mathrm{J} 1342+0928$, we correct for both the contrast and the CMB heating as prescribed by da Cunha et al. (2013), namely

$$
\begin{gathered}
f_{\mathrm{cmb}}=1-B_{\nu_{\mathrm{rest}}}\left(T_{\mathrm{cmb}, z}\right) / B_{\nu_{\text {rest }}}\left(T_{\mathrm{dust}, z}\right) \\
T_{\text {dust }, z}=\left(T_{\text {dust }}^{\beta+4}+T_{\mathrm{cmb}, z=0}^{\beta+4}\left[(1+z)^{\beta+4}-1\right]\right)^{\frac{1}{4+\beta}},
\end{gathered}
$$

where $T_{\text {dust }}$ is the intrinsic dust temperature the source would have at redshift of zero, $\beta$ is the same as in Equation (2), and $T_{\mathrm{cmb}, z}=2.73(1+z) \mathrm{K}=23.3 \mathrm{~K}$ is the CMB temperature at the redshift of $\mathrm{J} 1342+0928$.

We adopt the opacity coefficient of $\kappa_{\nu 0}=2.64 \mathrm{~m}^{2} \mathrm{~kg}^{-1}$ at $\nu_{0}=c /(125 \mu \mathrm{m})$ (Dunne et al. 2003). This parameter directly scales the dust mass and provides the dominant uncertainty on the dust mass estimate, which is at least a factor of two. All of our continuum measurements lie on the Rayleigh-Jeans tail and do not cover the peak of the dust SED; therefore, the fitting parameters are degenerate and the dust SED shape and its temperature cannot be fully constrained. Fixing the dust temperature to $47 \mathrm{~K}$, as is often assumed for quasar hosts in the literature (see, e.g., Beelen et al. 2006), results in $\beta=$ $1.85 \pm 0.3$ and $M_{\text {dust }}=(0.35 \pm 0.02) \times 10^{8} M_{\odot}$. The integral of the SED yields the FIR $(42.5-122.5 \mu \mathrm{m})$ and total infrared (TIR, $8-1000 \mu \mathrm{m})$ luminosities of $L_{\mathrm{FIR}}=(1.0 \pm 0.2) \times$ $10^{12} L_{\odot}$ and $L_{\mathrm{TIR}}=(1.5 \pm 0.3) \times 10^{12} L_{\odot}$. With this luminosity $\mathrm{J} 1342+0928$ can be classified as an ultra-luminous infrared galaxy (ULIRG, $\left.L_{\mathrm{TIR}}>10^{12} L_{\odot}\right)$. The TIR luminosity can be converted to the star formation rate (SFR) using the Kennicutt (1998) relation scaled to Chabrier (2003) initial mass function (IMF; yielding 1.7 times smaller SFR value than the Salpeter IMF) as

$$
\mathrm{SFR}\left[M_{\odot} \mathrm{yr}^{-1}\right]=10^{-10} L_{\mathrm{TIR}}\left[L_{\odot}\right],
$$

resulting in SFR of $150 \pm 30 M_{\odot} \mathrm{yr}^{-1}$ for $\mathrm{J} 1342+0928$. The calibration from Kennicutt \& Evans (2012) would give 14\% smaller values. A range of temperatures ${ }^{8}$ between 37 and $57 \mathrm{~K}$ yields $\beta=1.6-2.2, M_{\text {dust }}=(0.2-0.8) \times 10^{8} M_{\odot}, L_{\mathrm{FIR}}=$ $(0.8-1.3) \times 10^{12} L_{\odot}, L_{\mathrm{TIR}}=(1.0-2.2) \times 10^{12} L_{\odot}$, and $\mathrm{SFR}=(100-230) M_{\odot} \mathrm{yr}^{-1}$, consistent with previous estimates from NOEMA observations (Venemans et al. 2017b).

\subsection{Emission Lines}

Our observational spectral setups cover six atomic fine structure lines (from $\mathrm{C}, \mathrm{N}$, and $\mathrm{O}$ ), eight $\mathrm{CO}$ rotational lines, and multiple water and hydroxyl lines (see Table 2). Because of the low $\mathrm{S} / \mathrm{N}$ of all lines except the $[\mathrm{C} \mathrm{II}]_{158 \mu \mathrm{m}}$, we only analyze the integrated spectrum moment zero maps throughout. We show spectra of all detections and nondetections in Appendix B. Different atomic and molecular transitions trace different phases of the ISM and their line widths do not have to be equal. Nevertheless, we still adopt the same integration window for all potential lines as this provides the least biased measurement given the low $\mathrm{S} / \mathrm{N}$. Line luminosities were derived using equations given in Carilli \& Walter (2013; see also Solomon et al. 1997), namely

$$
\frac{L_{\text {line }}^{\prime}}{\mathrm{K} \mathrm{km} \mathrm{s}^{-1} \mathrm{pc}^{2}}=\frac{3.25 \times 10^{7}}{(1+z)^{3}} \frac{S_{\text {line }} \Delta v}{\mathrm{Jy} \mathrm{km} \mathrm{s}^{-1}}\left(\frac{D_{L}}{\mathrm{Mpc}}\right)^{2}\left(\frac{\nu_{\mathrm{obs}}}{\mathrm{GHz}}\right)^{-2},
$$

commonly used for molecular (specifically $\mathrm{CO}$ ) transitions, and

$$
\frac{L_{\text {line }}}{L_{\odot}}=1.04 \times 10^{-3} \frac{S_{\text {line }} \Delta v}{\mathrm{Jy} \mathrm{km} \mathrm{s}^{-1}}\left(\frac{D_{L}}{\mathrm{Mpc}}\right)^{2} \frac{\nu_{\mathrm{obs}}}{\mathrm{GHz}},
$$

often used when comparing lines and the underlying continuum.

\subsubsection{Fine Structure Lines}

We observe $[\mathrm{C} \mathrm{III}]_{158 \mu \mathrm{m}}, \quad[\mathrm{N} \mathrm{II}]_{205 \mu \mathrm{m}}, \quad[\mathrm{O} \mathrm{IIII}]_{88 \mu \mathrm{m}}, \quad$ and $[\mathrm{O} \mathrm{I}]_{146 \mu \mathrm{m}}$ fine structure lines at peak surface brightness $\mathrm{S} / \mathrm{N}$ of $12,4.1,5.7$, and 3.9, respectively. These measurements were taken from the moment zero maps integrated over $455 \mathrm{~km} \mathrm{~s}^{-1}$ $\left(336 \mathrm{~km} \mathrm{~s}^{-1}\right.$ in the case of $\left.[\mathrm{O} \mathrm{I}]_{146 \mu \mathrm{m}}\right)$, with subtracted continuum. We show these maps in Figure 3 and list measured aperture flux densities in Table 2.

The $[\mathrm{C} \mathrm{III}]_{158 \mu \mathrm{m}}$ observations were taken at a high resolution $(\approx 0$ !. 2$)$, where the emission is resolved into a morphologically complex structure, possibly a merger, which is discussed in the accompanying paper (Bañados et al. 2019). In this work we use only the total integrated emission to study relations between different lines and the underlying continuum averaged across the entire galaxy. The $[\mathrm{O} \mathrm{I}]_{146 \mu \mathrm{m}}$ emission line is also observed at high resolution. However, the surface brightness sensitivity

\footnotetext{
$\overline{8 \text { Larger temperature yields smaller } \beta \text { and }} M_{\text {dust }}$, but larger IR integrated luminosities.
} 
Table 2

Fine Structure and Molecular Line Measurements in J1342+0928

\begin{tabular}{|c|c|c|c|}
\hline Line & $\begin{array}{c}S_{\text {line }} \Delta v^{\mathrm{a}} \\
\left(\mathrm{Jy} \mathrm{km} \mathrm{s}^{-1}\right)\end{array}$ & $\begin{array}{c}L_{\text {line }} \\
\left(10^{8} L_{\odot}\right)\end{array}$ & $\left(10^{8} \mathrm{~K} \mathrm{~km} \mathrm{~s}^{L_{\text {line }}^{\prime}} \mathrm{pc}^{2}\right)$ \\
\hline$[\mathrm{C} \mathrm{I}]_{369 \mu \mathrm{m}}$ & $<0.074$ & $<0.41$ & $<24.0$ \\
\hline$[\mathrm{N} \mathrm{II}]_{205 \mu \mathrm{m}}$ & $<0.079$ & $<0.8$ & $<8.0$ \\
\hline$[\mathrm{C} \mathrm{III}]_{158 \mu \mathrm{m}}$ & $1.07 \pm 0.15$ & $14.0 \pm 1.9$ & $63.8 \pm 8.8$ \\
\hline$[\mathrm{O} \mathrm{I}]_{146 \mu \mathrm{m}}{ }^{\mathrm{b}}$ & $0.052-0.40$ & $0.73-5.7$ & $2.62-20$ \\
\hline$[\mathrm{N} \mathrm{III}]_{122 \mu \mathrm{m}}$ & $0.21 \pm 0.078$ & $3.55 \pm 1.3$ & $7.46 \pm 2.8$ \\
\hline$[\mathrm{O} \text { III }]_{88 \mu \mathrm{m}}$ & $1.14 \pm 0.41$ & $26.5 \pm 9.7$ & $21.2 \pm 7.7$ \\
\hline $\mathrm{CO}$ stack $^{\mathrm{c}}$ & $0.045 \pm 0.013^{\mathrm{d}}$ & $0.32 \pm 0.1$ & $9.0 \pm 2.7$ \\
\hline $\mathrm{CO}(7-6)$ & $<0.078$ & $<0.43$ & $<26.0$ \\
\hline $\mathrm{CO}(8-7)$ & $<0.08$ & $<0.51$ & $<20.0$ \\
\hline CO (10-9) & $<0.069$ & $<0.55$ & $<11.0$ \\
\hline CO $(11-10)$ & $<0.073$ & $<0.64$ & $<9.8$ \\
\hline CO (14-13) & $<0.24$ & $<2.7$ & $<20.0$ \\
\hline CO $(15-14)$ & $<0.12$ & $<1.4$ & $<8.3$ \\
\hline $\mathrm{CO}(16-15)$ & $<0.11$ & $<1.4$ & $<6.8$ \\
\hline $\mathrm{CO}(17-16)$ & $<0.097$ & $<1.3$ & $<5.5$ \\
\hline $\mathrm{OH}_{330 \mu \mathrm{m}}^{+}$ & $<0.073$ & $<0.46$ & $<19.0$ \\
\hline $\mathrm{OH}_{153.5 \mu \mathrm{m}}^{+}$ & $<0.095$ & $<1.3$ & $<5.4$ \\
\hline $\mathrm{OH}_{153.0 \mu \mathrm{m}}^{+}$ & $<0.097$ & $<1.3$ & $<5.4$ \\
\hline $\mathrm{OH}_{152.4 \mu \mathrm{m}}^{+}$ & $<0.092$ & $<1.2$ & $<5.1$ \\
\hline $\mathrm{OH}_{163.4 \mu \mathrm{m}}$ & $<0.084$ & $<1.1$ & $<5.4$ \\
\hline $\mathrm{OH}_{163.1 \mu \mathrm{m}}$ & $<0.095$ & $<1.2$ & $<6.0$ \\
\hline $\mathrm{H}_{2} \mathrm{O}_{260 \mu \mathrm{m}}^{312-221}$ & $<0.066$ & $<0.53$ & $<11.0$ \\
\hline $\mathrm{H}_{2} \mathrm{O}_{258 \mu \mathrm{m}}^{321-3_{12}}$ & $<0.059$ & $<0.47$ & $<9.4$ \\
\hline $\mathrm{H}_{2} \mathrm{O}_{175 \mu \mathrm{m}}^{303-212}$ & $<0.11$ & $<1.2$ & $<7.7$ \\
\hline $\mathrm{H}_{2} \mathrm{O}_{156 \mu \mathrm{m}}^{322-313}$ & $<0.038$ & $<0.5$ & $<2.2$ \\
\hline
\end{tabular}

Notes. Flux densities were measured inside a 2".6 diameter aperture, unless stated otherwise, and corrected for the residual. Upper limits correspond to $3 \sigma$ of the local rms noise.

${ }^{\mathrm{a}}$ In all cases $\Delta v=455 \mathrm{~km} \mathrm{~s}^{-1}$, except for [O I $]_{146 \mu \mathrm{m}}$ where $\Delta v=336 \mathrm{~km} \mathrm{~s}^{-1}$ due to availability of fewer channels.

${ }^{\mathrm{b}}$ Given insufficient surface brightness sensitivity of the higher resolution map we report a $3 \sigma$ upper limit on the aperture integrated emission, and a lower limit from the $3.9 \sigma$ significant single beam measurement.

c Stack of four CO lines in bands 3 and 4: CO (7-6), (8-7), (10-9), and (11 $-10)$.

${ }^{\mathrm{d}}$ Peak surface brightness and $\mathrm{rms}$ is reported (it is consistent with aperture integration within the errors, but has a higher $\mathrm{S} / \mathrm{N}$ ).

allows measurement of only the peak emission arising from the central region. This line lies on the edge of the sideband and is not fully recovered, and a smaller frequency range had to be used for integration across the line. Since the peak surface brightness of $[\mathrm{O} \mathrm{I}]_{146 \mu \mathrm{m}}$ is $3.9 \sigma$, we consider this line to be a tentative detection, and report a lower limit on the total emission measured from this single resolution element. Additionally, we provide a $3 \sigma$ upper limit on the integrated flux inside the aperture, where $\sigma$ is the uncertainty of the aperture integration. The $[\mathrm{N} \mathrm{II}]_{205 \mu \mathrm{m}}$ emission line is observed at lower resolution $(\sim 1$ !'25) and is spatially coincident with the continuum emission. We detect the $[\mathrm{O} \mathrm{III}]_{88 \mu \mathrm{m}}$ line at a resolution of $\sim 1^{\prime \prime}$ with a peak that is slightly offset $(0$. " 6$)$ from the central region and the underlying continuum; however, the line emission is still covered in its entirety by the aperture. Finally, no significant emission was observed for the $[\mathrm{C} \mathrm{I}]_{369 \mu} \mu \mathrm{m}$ and $[\mathrm{N} \mathrm{II}]_{205 \mu \mathrm{m}}$ lines and only upper limits can be obtained, which we set to $3 \sigma$ of the noise level.
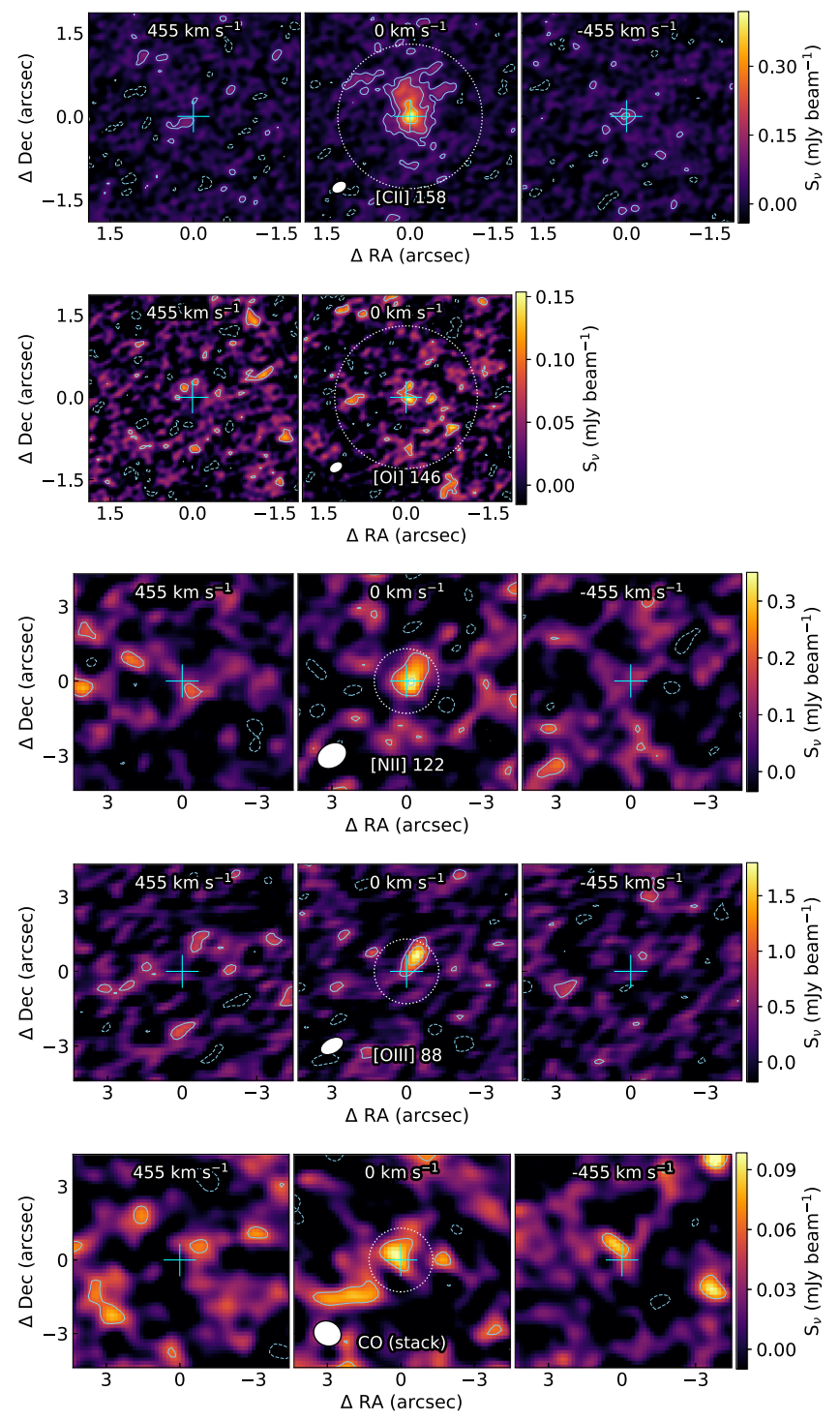

Figure 3. Cutouts of $\mathrm{J} 1342+0928$ showing the emission line in the central panel. Left and right panels demonstrate the absence of the continuum emission due to its successful subtraction. Each panel contains flux integrated over a width of $455 \mathrm{~km} \mathrm{~s}^{-1}\left(1.2 \times\right.$ FWHM of the $[\mathrm{C} \mathrm{III}]_{158 \mu \mathrm{m}}$ line $)$, with the exception of $[\mathrm{O} \mathrm{I}]_{146 \mu \mathrm{m}}$ where the central panel is $336 \mathrm{~km} \mathrm{~s}^{-1}$, due to its proximity to the sideband edge. Aperture used for flux integration is shown as the white dotted circle (diameter of 2 ." 6 ). The synthesized beam is shown in the lower left corner of the middle panel. Full (dashed) contours represent the $+(-) 2 \sigma, 4 \sigma$ and $8 \sigma$ emission significance. The rms values in the middle panel are, from top to bottom: $35,39,85,318$, and $30 \mu \mathrm{Jy}_{\text {beam }}{ }^{-1}$. The cross marks the dust continuum emission center in the high-resolution data (same as in Figure 1). The bottom three panels show a stack of four $\mathrm{CO}$ lines in bands 3 and 4 $\left(J_{\text {up }}=11,10,8\right.$, and 7).

\subsubsection{Molecular Lines}

We do not detect any individual molecular $\left(\mathrm{CO}, \mathrm{H}_{2} \mathrm{O}\right.$, and $\mathrm{OH}$ ) transitions, so we report their $3 \sigma$ upper limits ( $\sigma$ is the rms noise in the line map) in Table 2. Given the number of available lines that are covered with our observations, we have attempted to recover an average measurement through stacking. When the four lowest available $\mathrm{CO}$ transitions covered in bands 3 and 4 are stacked together (namely transitions 7-6, 8-7, 10-9, and $11-10$ ) we recover a $3.3 \sigma$ peak at the expected spatial and spectral coordinates, with a line flux of $0.045 \pm 0.013 \mathrm{Jy} \mathrm{km} \mathrm{s}-1$ 
extracted at the peak of the stacked emission. ${ }^{9}$ Data in the higher frequency bands have higher noise or poorer resolution, and the expected $\mathrm{CO}$ brightness is lower for higher $\mathrm{J}$ transitions, therefore we do not include them in our CO stack (we stacked them separately, but no significant detection was recovered). For the sake of convenience, we will treat the four line stack as a tentative $\mathrm{CO}(9-8)$ detection, because this transition frequency corresponds to the mean frequency of the stacked lines. However, the effective frequency of the stacked emission is strongly dependent on the actual shape of the CO SED, which is presently unknown.

We stacked two water lines in band 4 (260 and $258 \mu \mathrm{m})$, resulting only in a $3 \sigma$ upper limit on line flux density of $0.045 \mathrm{Jy} \mathrm{km} \mathrm{s}^{-1}$. The remaining two available water lines were observed at significantly different resolutions, and convolving all water lines to the same beam would result in a degraded signal quality.

\subsection{Properties of the ISM}

In the following sections we focus on specific lines and their ratios to derive various properties of the ISM in $\mathrm{J} 1342+0928$.

\subsubsection{The FIR Line Deficit}

An empirical observation showing a decrease in the $[\mathrm{C} \mathrm{III}]_{158 \mu \mathrm{m}}$ line-to-FIR continuum ratio with increasing dust temperature and IR luminosities is referred to as the line deficit (e.g., Malhotra et al. 1997; Díaz-Santos et al. 2013). The same trend was observed in other fine structure lines, thanks to systematic studies with Herschel (e.g., Díaz-Santos et al. 2017). The ISM conditions leading to such results are still under investigation and several scenarios have been proposed. These include a change in the ionization parameter, various optical depth effects, or galaxy compactness (for details see, e.g., Graciá-Carpio et al. 2011; Herrera-Camus et al. 2018; Rybak et al. 2019).

We report several fine structure line-to-FIR ratios of J1342 +0928 in Figure 4, along with a recent study of local luminous infrared galaxies (LIRGs; $L_{\mathrm{TIR}}=10^{10-11} L_{\odot}$ ) performed by Díaz-Santos et al. (2017). Measurements obtained on our $z=7.5$ quasar host galaxy are consistent within the scatter with trends observed in the local LIRGs.

\subsubsection{Photodissociation Regions (PDRs)}

The type of radiation field the ISM is subjected to divides its physical and chemical processes into two distinct regimes: the PDRs (also photon dominated regions) and the X-ray dissociation regions (XDRs; also X-ray dominated regions). The former implies the main source of energy are far-ultraviolet photons with energies between 6 and $13.6 \mathrm{eV}$ originating in $\mathrm{O}$ and B stars, and is therefore tied with star formation processes (see, e.g., Tielens \& Hollenbach 1985). In the latter regime, the gas is illuminated by hard X-ray radiation (energies above $1 \mathrm{keV}$ ), which penetrates much deeper into the molecular clouds before being absorbed (see, e.g., Maloney et al. 1996). Such hard radiation can originate in supermassive black hole accretion episodes and is therefore indicative of significant AGN activity.

\footnotetext{
We note that this value is consistent with the aperture integration within the errors, but has a higher $\mathrm{S} / \mathrm{N}$.
}

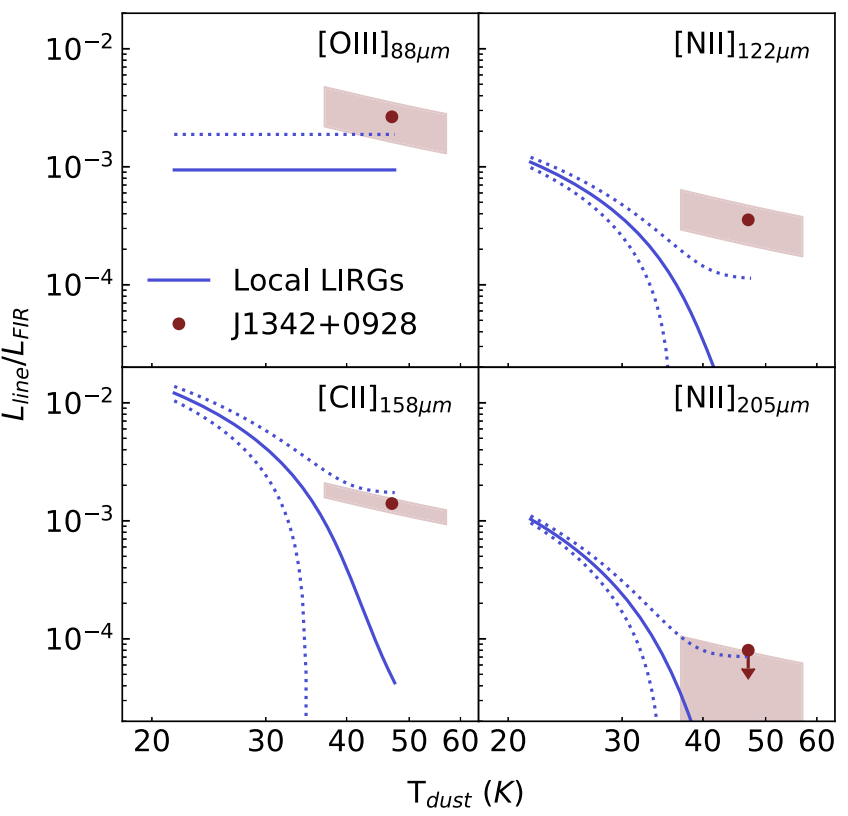

Figure 4. Ratio between various fine structure lines and the FIR (42.5 $122.5 \mu \mathrm{m}$ ) luminosities as a function of estimated dust temperature demonstrating the line deficit. Our J1342+0928 measurements are shown with a red point. The accompanying red shaded area encompasses the considered dust temperature range in the horizontal direction and the flux density measurement error in the vertical direction. The blue line shows the fit to the local trend in LIRGs, while the dotted lines outline its $1 \sigma$ dispersion (only the upper bound is constrained for the $[\mathrm{O} \mathrm{III}]_{88 \mu \mathrm{m}}$ case), as reported in Díaz-Santos et al. (2017).

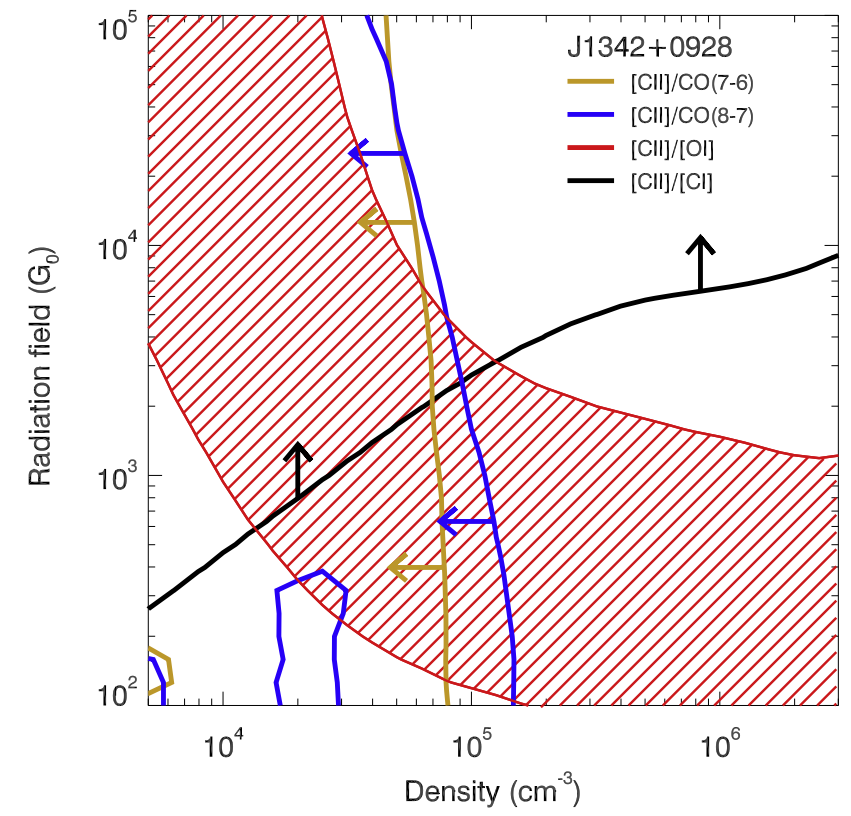

Figure 5. Constraints on the radiation field intensity (in units of the Habing flux, $G_{0}=1.6 \times 10^{-3} \mathrm{erg} \mathrm{cm}^{-2} \mathrm{~s}^{-1}$ ) and the density of the ISM in J1342 +0928 based on the PDR model grid from Meijerink et al. (2007). Colored lines correspond to contours in the parameter space defined by specific line luminosity ratios, namely $[\mathrm{C} \mathrm{III}]_{158 \mu \mathrm{m}} / \mathrm{CO}(7-6)>33$ (yellow), [C II $]_{158 \mu \mathrm{m} /}$ $\mathrm{CO}(8-7)>27$ (blue), $3<[\mathrm{C} \mathrm{III}]_{158 \mu \mathrm{m}} /[\mathrm{O} \text { I }]_{146 \mu \mathrm{m}}<19$ (red hatched area), and $[\mathrm{C} \mathrm{II}]_{158 \mu \mathrm{m}} /[\mathrm{C} \mathrm{I}]_{369 \mu \mathrm{m}}>34$ (black).

In order to tie our observations to the underlying physical properties, we make use of the PDR and XDR grid models developed by Meijerink \& Spaans (2005) and Meijerink et al. (2007). These models include various heating and cooling 


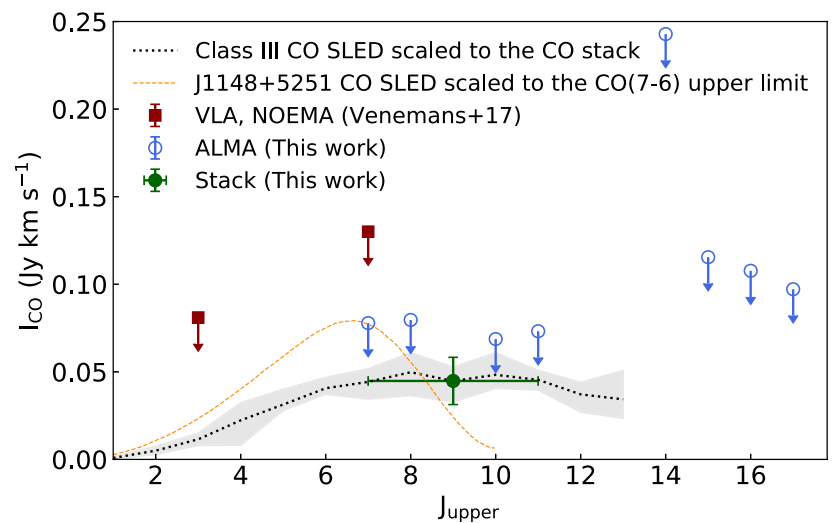

Figure 6. Constraints on the CO spectral line energy distribution of J1342 +0928 as a function of the rotational quantum number. The dotted black line shows the mean trends observed in local ULIRGs (see Rosenberg et al. 2015), scaled to our stacked measurement (gray shaded area shows the scatter). The dashed orange line shows the CO SLED model of a $z=6.4$ quasar (Stefan et al. 2015) scaled to our $\mathrm{CO}(7-6)$ upper limit.

processes and chemical reactions that reproduce emission line luminosities as a function of physical parameters, such as the radiation field and density of the ISM. For the PDR calculations, we may consider only emission arising in the neutral medium. The $[\mathrm{C} \mathrm{III}]_{158 \mu \mathrm{m}}$ can originate in the ionized medium as well; however, we will show in Section 3.3.5 that this fraction can be constrained to less than $25 \%$. Since we can only obtain an upper limit, we do not correct for the emission fraction arising in ionized medium, and assume that the entire $[\mathrm{C} \mathrm{II}]_{158 \mu \mathrm{m}}$ emission is dominated by the PDR.

The line luminosity ratio $[\mathrm{C} \mathrm{II}]_{158 \mu \mathrm{m}} /[\mathrm{C} \mathrm{I}]_{369 \mu \mathrm{m}}$ is useful for discerning between the two main regimes (PDR and XDR) as the ratio is expected to be below 6 in XDRs regardless of ISM density or the radiation field (see Figure 6 in Venemans et al. 2017c). Although we do not detect the $[\mathrm{C} \mathrm{I}]_{369 \mu \mathrm{m}}$ line, its upper limit sets a lower limit on the line luminosity ratio $[\mathrm{C} \mathrm{II}]_{158 \mu \mathrm{m}} /[\mathrm{C} \mathrm{I}]_{369 \mu \mathrm{m}}>34$, which rules out XDR as the dominant regime in $\mathrm{J} 1342+0928$. We can therefore assume pure PDR models for the subsequent analysis. In PDRs, the ratio of $[\mathrm{C} \mathrm{III}]_{158 \mu \mathrm{m}}$ and $\mathrm{CO}$ emission lines is strongly dependent on the density, and we can use our limits listed in Table 2 to define an area in the density versus radiation field parameter space, which $\mathrm{J} 1342+0928$ occupies. As shown in Figure 5, PDR model parameters, which are derived from our limits, suggest densities of $\lesssim 5 \times 10^{4} \mathrm{~cm}^{-3}$ and radiation field intensity of $\gtrsim 10^{3} G_{0}$, where $G_{0}$ is the Habing flux $\left(G_{0}=1.6 \times 10^{-3} \mathrm{erg} \mathrm{cm}^{-2} \mathrm{~s}^{-1}\right)$. For comparison, similar ISM studies performed in five different $z>6$ quasars (see Wang et al. 2016; Venemans et al. 2017a, and Yang et al. 2019) find densities higher than $10^{5} \mathrm{~cm}^{-3}$, and radiation field intensities of the order of $\sim 10^{3} G_{0}$. On the other hand, several studies based on $\mathrm{CO}$ excitation ladders suggest densities in the range of $10^{4-5} \mathrm{~cm}^{-3}$ (see Weiß et al. 2007; Riechers et al. 2009).

\subsubsection{Carbon Mass}

The measured upper limit of the $[\mathrm{C} \mathrm{I}]_{369 \mu \mathrm{m}}$ emission line can provide constraints on the atomic carbon content of the galaxy. Following Weiß et al. (2003, 2005) we can estimate the mass of
Table 3

The Total Gas Mass of J1342+0928 Estimated Using Different Proxies (see Section 3.3.4 for Details)

\begin{tabular}{lcc}
\hline \hline Proxy and Extrapolation & $\begin{array}{c}\text { Gas Mass } \\
\left(10^{8} M_{\odot}\right)\end{array}$ & $\begin{array}{c}\text { Gas-to-dust } \\
\text { ratio }\end{array}$ \\
\hline CO to FIR continuum correlation & 80 & 230 \\
CO $(7-6)$ with J1148+5251 SLED & $<35$ & $<100$ \\
CO stack with Class III SLED & 11 & 30 \\
{$[\mathrm{C} \mathrm{III}]_{158} \mu \mathrm{m}$ calibration } & 420 & 1200 \\
\hline
\end{tabular}

Note. Conversion factor of $\alpha_{\mathrm{CO}}=0.8 M_{\odot}\left(\mathrm{K} \mathrm{km} \mathrm{s}^{-1} \mathrm{pc}^{2}\right)^{-1}$ is used for all CO measurements.

the neutral carbon as

$$
\frac{M_{[\mathrm{C} \mathrm{I}]}}{M_{\odot}}=4.566 \times 10^{-4} Q\left(T_{\mathrm{ex}}\right) \frac{1}{5} e^{T_{2} / T_{\mathrm{ex}}} \frac{L_{[\mathrm{C}]_{369} \mu \mathrm{m}}^{\prime}}{\mathrm{K} \mathrm{km} \mathrm{s}^{-1} \mathrm{pc}^{2}},
$$

where $Q\left(T_{\mathrm{ex}}\right)=1+3 e^{-T_{1} / T_{\mathrm{ex}}}+5 e^{-T_{2} / T_{\mathrm{ex}}}$ is the partition function, excitation energies of two different carbon transitions are $T_{1}=23.6 \mathrm{~K}$ and $T_{2}=62.5 \mathrm{~K}$, and the excitation temperature is assumed to be $T_{\mathrm{ex}}=30 \mathrm{~K}$ (see Walter et al. 2011). Resulting atomic carbon mass limit is $M_{[\mathrm{CI}]}<5.3 \times 10^{6} M_{\odot}$. Walter et al. (2011) derived an atomic carbon to molecular hydrogen abundance of $\mathrm{X}[\mathrm{C} \mathrm{I}] / \mathrm{X}\left[\mathrm{H}_{2}\right]=M_{[\mathrm{C} \mathrm{I}]} /\left(6 M_{\mathrm{H}_{2}}\right)=(8.4 \pm 3.5) \times$ $10^{-5}$ using a sample of $z>2$ submillimeter and quasar host galaxies. If we apply the same scaling relation to $\mathrm{J} 1342+0928$ we obtain $M_{\mathrm{H}_{2}}<10^{10} M_{\odot}$ implying a gas-to-dust ratio upper limit of $<250$.

For completeness, we mention here the ionized carbon mass of $M_{[\mathrm{C} \mathrm{II}]}=4.9 \times 10^{6} M_{\odot}$ as reported by Venemans et al. (2017b), emitted from the outer layers of the PDR assuming $T_{\text {ex }}=100 \mathrm{~K}$ (temperature range from 75 to $200 \mathrm{~K}$ would give a $20 \%$ difference on the estimated mass).

\subsubsection{Gas Mass}

In this section we constrain the total molecular gas mass of $\mathrm{J} 1342+0928$ employing a variety of methods. They are listed in Table 3 and explained below. The most common approach involves using the $\mathrm{CO}$ molecule as an $\mathrm{H}_{2}$ tracer. In order to obtain the gas mass from the $\mathrm{CO}$ line emission, we must assume a CO-to- $\mathrm{H}_{2}$ (light-to-mass) conversion factor $\alpha_{\mathrm{CO}}$ (see Bolatto et al. 2013). The scaling is linear with line luminosity and is defined as $M_{\mathrm{H}_{2}}\left[M_{\odot}\right]=\alpha_{\mathrm{CO}} L_{\mathrm{CO}(1-0)}^{\prime}\left[\mathrm{K} \mathrm{km} \mathrm{s}^{-1} \mathrm{pc}^{2}\right]$. We employ a value of $\alpha_{\mathrm{CO}}=0.8 M_{\odot}\left(\mathrm{K} \mathrm{km} \mathrm{s}^{-1} \mathrm{pc}^{2}\right)^{-1}$ (see Downes \& Solomon 1998), which is often used. However, we note that a broader range of $\alpha_{\mathrm{CO}}=0.3-2.5 M_{\odot}\left(\mathrm{K} \mathrm{km} \mathrm{s}^{-1} \mathrm{pc}^{2}\right)^{-1}$ is recommended for ULIRGs (see Bolatto et al. 2013). The $\alpha_{\mathrm{CO}}$ factor is highly uncertain largely due to unknown average surface density of the giant molecular clouds and the kinetic temperature of the gas. To use this scaling relation, we first need to estimate the (unobserved) luminosity of the $\mathrm{CO}$ transmission of the rotational ground state.

One approach in estimating the $\mathrm{CO}(1-0)$ emission would be to use its empirical correlation with the FIR continuum, $\log \left(L_{\mathrm{FIR}}\left[L_{\odot}\right] / L_{\mathrm{CO}(1-0)}^{\prime}\left[\mathrm{K} \mathrm{km} \mathrm{s}^{-1} \mathrm{pc}^{2}\right]\right) \sim 2$ with a scatter of 0.5 dex, as shown in Figure 7 of Carilli \& Walter (2013). This correlation would imply $L_{\mathrm{CO}(1-0)}^{\prime} \sim 100 \times 10^{8} \mathrm{~K} \mathrm{~km} \mathrm{~s}^{-1} \mathrm{pc}^{2}$, and thus a high gas-to-dust mass ratio of $\sim 230$. 
We have a significant number of $\mathrm{CO}$ upper limits as well as a tentative stack detection, as shown in Figure 6. If the $\mathrm{CO}(9-8)$ is thermalized it would imply $L_{\mathrm{CO}(1-0)}^{\prime}=L_{\mathrm{CO}(9-8)}^{\prime}$. A correction to this simplified model involves usage of a $\mathrm{CO}$ spectral line energy distribution (SLED) of a high-redshift quasar. Stefan et al. (2015) employed large velocity gradients to model the CO SLED of J114816.64+525150.3, a $z=6.4$ quasar, using four observed $\mathrm{CO}$ transitions up to $J_{\text {up }}=7$. This model is also shown in Figure 6, scaled to our $\mathrm{CO}(7-6)$ upper limit. The model extrapolation beyond $J_{\text {up }} \geqslant 8$ is consistent with our stacked measurement within $2 \sigma$ and predicts $L_{\mathrm{CO}(1-0)}^{\prime}<$ $44 \times 10^{8} \mathrm{~K} \mathrm{~km} \mathrm{~s}^{-1} \mathrm{pc}^{2}, M_{\mathrm{H}_{2}}<35 \times 10^{8} M_{\odot}$, and a gas-todust ratio of $<100$, based on our $\mathrm{CO}(7-6)$ upper limit.

We can also consider the CO (SLEDs) of the local ULIRGs. In Figure 6 we show the Class III CO SLED, which has a turnover at higher $J$ levels, as reported by Rosenberg et al. (2015), scaled to our stack measurement. If we employ the CO SLED of ARP 299, a merger induced starburst belonging to the abovementioned Class III group, the line flux of the $\mathrm{CO}(1-0)$ transition is $\sim 50$ times weaker than the $\mathrm{CO}(9-8)$ transition, which from Equation (7) implies $L_{\mathrm{CO}(1-0)}^{\prime} \sim 1.5 L_{\mathrm{CO}(9-8)}^{\prime}=$ $13.5 \times 10^{8} \mathrm{~K} \mathrm{~km} \mathrm{~s}^{-1} \mathrm{pc}^{2}, M_{\mathrm{H}_{2}}=(11 \pm 3) \times 10^{8} M_{\odot}$ and a somewhat small gas-to-dust mass ratio of $\sim 30$.

Zanella et al. (2018) suggests to use the $[\mathrm{C} \mathrm{II}]_{158 \mu \mathrm{m}}$ emission as a tracer of molecular gas mass via the conversion factor of $\left.M_{\mathrm{H}_{2}} / L_{[\mathrm{C} \mathrm{II}}\right]_{158 \mu \mathrm{m}}=\alpha_{[\mathrm{C} \mathrm{II}]} \sim 30 M_{\odot} / L_{\odot}$, with a standard deviation of $0.2 \mathrm{dex}$, based on a sample of main-sequence galaxies at $z \sim 2$. These authors report that the calibration is largely independent of the phase gas metallicity and the starburst behavior of the galaxy. When applied to $\mathrm{J} 1342+0928$ we obtain molecular mass of $M_{\mathrm{H}_{2}} \sim 4 \times 10^{10} M_{\odot}$. This would imply very large gas-to-dust ratios of $\sim 1200$ and is not consistent with our nondetection of neutral carbon (see Section 3.3.3). Although large uncertainties are involved in the estimates, our upper limits and the tentative stack detection, paired with different models of the CO SLEDs, do not favor such a large gas-to-dust ratio. For comparison, standard gas-todust ratios observed in nearby galaxies are around $\sim 100$ (e.g., Sandstrom et al. 2013).

\subsubsection{Ionized Gas}

Due to different ionization energies of the fine structure lines (for $\mathrm{C}^{+}, \mathrm{O}^{+}, \mathrm{N}^{+}$, and $\mathrm{O}^{++}$they are $11.3,13.6,14.5$, and $35 \mathrm{eV}$ respectively), and their critical densities, ${ }^{10}$ line ratios can further constrain the properties of the ISM, specifically the H II regions. The $[\mathrm{N} \mathrm{II}]_{205} \mu \mathrm{m}$ lines originate exclusively in the ionized medium due to its ionization energy being higher than that of hydrogen $(13.6 \mathrm{eV})$. The model provided by Oberst et al. (2006), see their Figure 2, which assumes an electron impact excitation and electron temperature of $8000 \mathrm{~K}$, allows us to use our measurement of $[\mathrm{N} \mathrm{II}]_{122 \mu \mathrm{m}} /[\mathrm{N} \mathrm{II}]_{205 \mu \mathrm{m}}>4.4$ to set a lower limit on the electron density of $n_{e}>180 \mathrm{~cm}^{-3}$, hence the $\mathrm{N}^{+}$emission is dominated by the $\mathrm{H}$ II regions and not the diffuse ISM.

Carbon has a lower ionization energy than hydrogen allowing the $[\mathrm{C} \mathrm{III}]_{158 \mu \mathrm{m}}$ emission line to arise in neutral and ionized medium. Both transitions $[\mathrm{C} \mathrm{II}]_{158 \mu \mathrm{m}}$ and $[\mathrm{N} \mathrm{III}]_{205 \mu \mathrm{m}}$ have similar critical densities, $40 \mathrm{~cm}^{-3}$ and $44 \mathrm{~cm}^{-3}$, respectively, implying that their line luminosity ratio will depend solely on

\footnotetext{
${ }^{10}$ De-excitation emission rates are equal to collision rates at the critical density.
}

their abundance ratio. This fact allows us to use their line ratio in order to disentangle the fraction of emission arising in different phases of the ISM. At the limit obtained above for the electron density $\left(n_{e}>180 \mathrm{~cm}^{-3}\right)$, the expected value of the carbon to nitrogen line ratio inside the ionized medium, according to Oberst et al. (2006), is $3 \lesssim[\mathrm{C} \mathrm{II}]_{158 \mu \mathrm{m}} /[\mathrm{N} \mathrm{III}]_{205 \mu \mathrm{m}}<4.3$. From this ratio, and our $[\mathrm{N} \mathrm{III}]_{205 \mu \mathrm{m}}$ luminosity limit of $<0.8 \times 10^{8} L_{\odot}$, we can derive an upper limit on the $[\mathrm{C} \mathrm{II}]_{158 \mu \mathrm{m}}$ emission arising from the ionized medium of $[\mathrm{C} \mathrm{II}]_{158}^{\text {ion }}<3.4 \times 10^{8} L_{\odot}$. This accounts for less than $25 \%$ of the entire $14 \times 10^{8} L_{\odot}$ observed [C III $]_{158 \mu \mathrm{m}}$ emission.

Following Ferkinhoff et al. (2011), assuming high density and high temperature limit so that all nitrogen is singly ionized (valid around B2 to 08 stars), we can calculate the minimum $\mathrm{H}^{+}$mass required to produce the observed $\mathrm{N}^{+}$luminosity as

$$
M\left(\mathrm{H}^{+}\right)=\frac{\left.L_{[\mathrm{N} \mathrm{II}}\right]_{122 \mu \mathrm{m}}}{\frac{g_{2}}{g_{t}} A_{21} h \nu_{21}} \frac{m_{\mathrm{H}}}{\chi\left(\mathrm{N}^{+}\right)},
$$

where parameters are statistical weight of the emission line $g_{2}=5$, partition function $g_{t}=9$, Einstein coefficient $A_{21}=$ $7.5 \times 10^{-6} \mathrm{~s}^{-1}$, relative abundance of nitrogen compared to hydrogen $\chi\left(\mathrm{N}^{+}\right)=9.3 \times 10^{-5}$ (taken from Savage \& Sembach 1996), hydrogen atom mass $m_{\mathrm{H}}$, Planck constant $h$, and the frequency of the $122 \mu \mathrm{m}$ line transition $\nu_{21}$. Inserting the luminosity value of $L_{[\mathrm{N} \mathrm{II}]_{122 \mu \mathrm{m}}}=3.55 \times 10^{8} L_{\odot}($ from Table 2$)$ yields $M_{\min }\left(\mathrm{H}^{+}\right)=1.8 \times 10^{8} M_{\odot}$. When compared to the molecular gas mass derived from the $\mathrm{CO}(7-6)$ upper limit we obtain a lower limit on the gas ionization percentage $M\left(\mathrm{H}^{+}\right) / M\left(\mathrm{H}_{2}\right)>4 \%$, while the estimate from the $\mathrm{CO}$ stack measurement would yield an even greater value of $\sim 16 \%$. For comparison, the ionized gas fraction is observed to be less than $<1 \%$ in nearby galaxies (see Figure 3 in Ferkinhoff et al. 2011 based on Brauher et al. 2008 data), while larger ionization percentages point to the increased SFR surface density ( 100-1000 $\left.M_{\odot} \mathrm{yr}^{-1} \mathrm{pc}^{-2}\right)$.

A similar approach can be utilized for the $\mathrm{O}^{++}$line, as described by Ferkinhoff et al. (2010). We can insert into Equation (9) values valid for the $[\mathrm{O} \mathrm{III}]_{88} \mu \mathrm{m}$ line, namely $\left.L_{[\mathrm{O} \mathrm{III}}\right]_{88 \mu \mathrm{m}}=26.5 \times 10^{8} L_{\odot}, \quad g_{2}=3, \quad g_{t}=9, \quad A_{21}=2.7 \times$ $10^{-5} \mathrm{~s}^{-1}, \chi\left(\mathrm{O}^{++}\right)=5.9 \times 10^{-4}$ (from Savage \& Sembach 1996), thus obtaining $M_{\min }\left(\mathrm{H}^{+}\right)=0.7 \times 10^{8} M_{\odot}$. We do not expect all oxygen to be in the doubly ionized state (see Ferkinhoff et al. 2010), so the minimum ionized gas we report here is strictly a lower limit and it can easily be an order of magnitude higher.

Further investigation of ISM physical properties via emission line ratios is available with the Cloudy spectral synthesis code (Ferland et al. 2017). The model computes line strengths on a grid of changing ISM conditions and we defer the details of the simulation and its setup to an upcoming paper by R. Decarli et al. 2019, (in preparation). Intensity of the radiation field can be estimated from the observed line ratio $[\mathrm{O} \mathrm{III}]_{88 \mu \mathrm{m}} /[\mathrm{N} \mathrm{III}]_{122 \mu \mathrm{m}}=7.5 \pm 4.0$. This is possible due to the fact that both ions have similar critical densities, but different ionization energies. Assuming that stars with effective temperature of $\sim 40,000 \mathrm{~K}$ are responsible for the ionization radiation, the model predicts intensity of the radiation field of $\log U \sim-2.5$, where $U$ is the ionization parameter defined as a ratio between the ionizing photon density and the total 
hydrogen density (see, e.g., Draine 2011): $U=n(h \nu>$ $13.6 \mathrm{eV}) / n_{\mathrm{H}}$. An equivalent way to write the ionization parameter is $U=\Phi /\left(n_{\mathrm{H}} c\right)$, where $\Phi$ is the flux of the hydrogen ionizing photons, and $c$ is the speed of light.

Assuming that the star formation is the main driver of observed atomic line emissions, we can use findings reported in De Looze et al. (2014) to calculate the SFR of J1342+0928 using FIR fine structure lines. For high-redshift galaxies $(z>0.5)$ these authors report the following calibration:

$$
\log \left(\mathrm{SFR} / M_{\odot}\right)=\beta+\alpha \log \left(L_{\text {line }} / L_{\odot}\right),
$$

where the parameters are $\alpha=1$ and $\beta=-6.89$ for the $[\mathrm{O} \mathrm{III}]_{88 \mu \mathrm{m}}$ emission line, with a dispersion of $0.46 \mathrm{dex}$. This calibration yields $\mathrm{SFR}=340 M_{\odot} \mathrm{yr}^{-1}$. Similarly, for $[\mathrm{C} \mathrm{II}]_{158 \mu \mathrm{m}}(\alpha=1.18$ and $\beta=-8.52$, dispersion 0.4 dex $)$ we obtain SFR $=180 M_{\odot} \mathrm{yr}^{-1}$. Both of these values agree within the uncertainties with the SFR inferred from the dust continuum.

As is evident from Figure 3, the peak of the $[\mathrm{O} \mathrm{III}]_{88 \mu \mathrm{m}}$ emission is offset by 0 ! 6 from the peak of the underlying continuum and peaks of other detected lines. Although this might be attributed to possible outflows (see, e.g., Bischetti et al. 2017), the low $\mathrm{S} / \mathrm{N}$ of our detection, and the fact that the separation is less than one resolution element, does not allow for a robust interpretation. Theoretical studies performed by Katz et al. $(2017,2019)$ predict that $[\mathrm{O} \mathrm{III}]_{88 \mu \mathrm{m}}$ would be at an offset from $[\mathrm{C} \mathrm{II}]_{158 \mu \mathrm{m}}$, because it arises from a medium with higher temperatures and ionization parameter. They also predict that $[\mathrm{N} \mathrm{II}]_{205} \mu \mathrm{m}$ emission would be slightly more extended compared to the $[\mathrm{C} \mathrm{II}]_{158 \mu \mathrm{m}}$ emission. Deeper data, preferably at higher resolution comparable to the $[\mathrm{C} \mathrm{III}]_{158 \mu \mathrm{m}}$ data, is required to investigate this potential offset further. Finally, we report oxygen-to-carbon line luminosity ratio of $[\mathrm{O} \mathrm{III}]_{88 \mu \mathrm{m}} /[\mathrm{C} \mathrm{III}]_{158 \mu \mathrm{m}}=1.9 \pm 0.7$, similar to other recent high-redshift observations (see Hashimoto et al. 2018; Walter et al. 2018).

\subsubsection{Water}

Water line emission originates in the warm dust $(40-70 \mathrm{~K})$ regions where densities are of the order of $10^{5-6} \mathrm{~cm}^{-3}$, and higher rotational CO lines $\left(J_{\text {up }}>8\right)$ are also common (see Liu et al. 2017). We do not detect any significant emission from $\mathrm{H}_{2} \mathrm{O}, \mathrm{OH}$, and $\mathrm{OH}^{+}$species within 10 possible line candidates that lie inside our observed frequency ranges. Water lines $3_{12}-2_{21}$ at $260 \mu \mathrm{m}$ and $3_{03}-2_{12}$ at $175 \mu \mathrm{m}$ show tentative detections; however, the first one could be dominated by the neighboring $\mathrm{CO}(10-9)$ line, while the second one shows an excess in the spectrum, but a prominent negative peak at the required central frequency (see Appendix B). Our PDR measurements (see Section 3.3.2) point toward lower densities, thus nondetection of water lines is consistent with our findings determined from $\mathrm{CO}$ nondetections.

\subsubsection{Metallicity}

Due to the high dust content and large SFRs, we expect that the ISM of $\mathrm{J} 1342+0928$ has already been enriched by the yields of supernovae. The metallicity of a galaxy is usually determined from optical line ratios, which are severely affected by dust extinction, and are not accessible to ground instruments for high-redshift objects. The FIR emission lines can provide an alternative method to measure metallicity, once it is properly calibrated (see, e.g., Pereira-Santaella et al. 2017). The $\mathrm{N}^{++}$ and $\mathrm{O}^{++}$ions are especially interesting for this study due to their similar ionization structure. We use the calibration reported by Rigopoulou et al. (2018), see their Figure 5, and assume that the ionization parameter is $\log U=-2.5$. From the $[\mathrm{O} \mathrm{III}]_{88 \mu \mathrm{m}} /[\mathrm{N} \mathrm{II}]_{122 \mu \mathrm{m}}=7.5 \pm 4$ line ratio we derive gas-phase metallicities in the $\mathrm{H}$ II region of $Z_{\text {gas }}=1.3_{-0.1}^{+0.3} Z_{\odot}$, where $Z_{\odot}$ is the value for the solar neighborhood. A range of $\log U$ between -2 and -3 would yield metallicities of $0.7-2.0 Z_{\odot}$, with line flux errors folded inside.

Multiple studies of high-redshift quasars performed using the optical spectra derive metallicities consistent with values observed in the solar neighborhood (see, e.g., Kurk et al. 2007; De Rosa et al. 2011; Mazzucchelli et al. 2017). These measurements were obtained from the $\mathrm{Mg}$ II / Fe II line ratio measured in the broad line region, at spatial scales of $\lesssim 1 \mathrm{kpc}$. On the other hand, our measurements, also pointing to solar like metallicities, are averaged across the entire galaxy host.

Rémy-Ruyer et al. (2014) have shown that the metallicity is an important contributor to the observed gas-to-dust ratio. These authors report that the observed gas-to-dust ratio in their sample is around $\sim 100$ for metallicities around solar, with a clear negative correlation between the two. Our findings of the rich metal content in the host galaxy of $\mathrm{J} 1342+0928$ are therefore in agreement with the low estimates on its gas-to-dust ratio derived from the $\mathrm{CO}$ limits (or the $\mathrm{CO}$ stack).

\section{Conclusions}

We have presented ALMA observations of the dust continuum and the ISM of the host galaxy J1342+0928, the most distant quasar known to date. The quasar was observed at $z=7.54$ when the universe was only 680 millions years old. With eight spectral setups positioned between 93.5 and $412 \mathrm{GHz}$, we constrain the Rayleigh-Jeans tail of the dust continuum and show that a modified blackbody with the canonical dust temperature of $47 \mathrm{~K}$ fits the data well. At the same time we narrow the dust spectral emissivity coefficient to $\beta=1.85 \pm 0.3$, derive the dust mass of $M_{\text {dust }}=0.35 \times$ $10^{8} M_{\odot}$ and a high SFR of $\sim 150 M_{\odot} \mathrm{yr}^{-1}$.

With detections of atomic fine structure lines $[\mathrm{C} \mathrm{III}]_{158 \mu \mathrm{m}}$, $[\mathrm{N} \mathrm{III}]_{205 \mu \mathrm{m}}, \quad[\mathrm{O} \mathrm{III}]_{88 \mu \mathrm{m}}$; limits on $[\mathrm{C} \mathrm{I}]_{369 \mu \mathrm{m}}, \quad[\mathrm{N} \mathrm{III}]_{205} \mu \mathrm{m}$, $[\mathrm{O} \mathrm{I}]_{146 \mu \mathrm{m}}$; and multiple $\mathrm{CO}$ lines (with a tentative stack detection) we derived the following main results. Observed line deficits in $\mathrm{J} 1342+0928$ are comparable to local ULIRGs. We do not see evidence of X-ray dominated regions, and photodissociation region-only model implies low gas densities $\left(\lesssim 5 \times 10^{4} \mathrm{~cm}^{-3}\right)$ and strong radiation fields $\left(\gtrsim 10^{3} G_{0}\right)$. The $\mathrm{CO}$ data hints at lower gas-to-dust ratios, less than 100. The $[\mathrm{C} \mathrm{II}]_{158 \mu \mathrm{m}}$ emission originates predominantly inside the neutral medium, and there is evidence of possible spatial offset between $[\mathrm{C} \mathrm{III}]_{158 \mu \mathrm{m}}$ and $[\mathrm{O} \mathrm{IIII}]_{88} \mu \mathrm{m}$ emission. However, deeper data is required to confirm and interpret this offset. A limit on the ratio of the two $\mathrm{N}^{++}$emission lines imply high electron densities of $n_{e}>180 \mathrm{~cm}^{-3}$. We also find high percentages ( $>4 \%$, possibly $\sim 16 \%$ ) of ionized to molecular hydrogen, indicating large SFR surface densities. We detect no water lines consistent with upper limits on $\mathrm{CO}$ lines and the derived density of the medium. Finally, we estimate that the metallicities are consistent with solar values, supporting the view of $\mathrm{J} 1342+0928$ as a galaxy extremely rich in dust and metals, despite the early epoch it is located in. 
In this study of the ISM of a galaxy in the epoch of reionization, we demonstrate the wide range of investigations that are possible with ALMA observations of FIR emission lines. We have shown that such an analysis benefits greatly from observations of multiple lines arising in different states of the ISM. The coming years will provide more high-redshift candidates, and ALMA follow-ups targeting several FIR emission lines in these objects will lead to a deeper understanding of the ISM at the epoch of reionization.

We thank Dominik Riechers for helpful discussions and suggestions. Ml.N., F.W., B.V., and Ma.N. acknowledge support from the ERC Advanced Grant 740246 (Cosmic Gas). This paper makes use of the following ALMA data: ADS/JAO.ALMA\#2017.1.00396.S. ALMA is a partnership of ESO (representing its member states), NSF (USA) and NINS (Japan), together with NRC (Canada), NSC and ASIAA (Taiwan), and KASI (Republic of Korea), in cooperation with the Republic of Chile. The Joint ALMA Observatory is operated by ESO, AUI/NRAO, and NAOJ. This research made use of Astropy, ${ }^{11}$ a community-developed core Python package for Astronomy (Astropy Collaboration et al. 2013; PriceWhelan et al. 2018), and Matplotlib (Hunter 2007).

\section{Appendix A}

\section{Measuring Resolved Emission in Interferometric Maps}

The difficulty of properly interpreting the area of the synthesized beam in interferometric maps can have a detrimental effect on the accuracy of the measured flux density, especially for significantly resolved emission. A Fourier transform of measured visibilities is called a dirty map, where each pixel has a unit of jansky per dirty beam. The integral under the entire dirty beam is zero, because an integral of a single sine wave is zero, and the dirty beam is just a sum of various sine waves. During the cleaning process, the dirty beam is deconvolved from the map and replaced with a Gaussian beam with a properly defined integral. The cleaning process progresses down to some chosen threshold, and the final cleaned interferometric map is then a combination of the residual given in original units of jansky per dirty beam, and a cleaned component in units of jansky per clean beam (chosen to be a Gaussian). The end result is that units in the map are ill defined, and if the observed source is spread over multiple beams, the cumulative effect can be significant.

Several choices are available to tackle this problem. The first one is to model the source using visibilities directly, without invoking the Fourier transform and going into the image plane. This works well for objects exhibiting some sort of symmetry, but is less useful for morphologically complex systems such as mergers, where a lot of details are hidden in the data coming from high-resolution/longer baselines. The second choice is to clean very deep, which comes at a cost of treating every noise peak (both positive and negative) as emission. The third choice, which we employ in this paper, involves estimating the proper area of the dirty beam in the region of interest, as the dirty beam area is zero when integrated across all space, but is different from zero in a smaller region. This method is called the residual scaling and is outlined in Appendix A.2 of Jorsater \& van Moorsel (1995), see also Walter \& Brinks (1999) and Walter et al. (2008). We summarize it here briefly.

We can consider the true flux $G$ of a source as a sum of cleaned flux $C$ in proper jansky per beam units, and the residual $R$, which has to be scaled to proper units in order to make the sum viable: $G=C+\epsilon R$. The parameter $\epsilon$ corresponds to the clean beam to dirty beam area ratio. If the same map is cleaned down to two different thresholds, we have $G=C_{1}+\epsilon$ $R_{1}=C_{2}+\epsilon R_{2}$, because the true flux must be recovered independent of the cleaning threshold. We can also choose not to clean, in which case $C_{2}=0$ and $R_{2}$ is the dirty map. Solving equations in this scenario yields $\epsilon=C_{1} /\left(R_{2}-\mathrm{R}_{1}\right)$ and $G=\epsilon$ $R_{2}$. These calculations are performed within some aperture, and use the dirty map $R_{2}$, the clean flux map $C_{1}$ and the residual map $R_{1}$ to measure the flux density $G$. A drawback of the residual scaling method arises in situations when $R_{1} \approx R_{2}$, or when there is not enough clean flux $C_{1}$, and the solution becomes numerically unstable.

In Figure 7 we demonstrate this method on our highresolution $(\sim 0$ ". 2$)$ observations of $\mathrm{J} 1342+0928$ at $231.5 \mathrm{GHz}$ and the $[\mathrm{C} \mathrm{II}]_{158 \mu \mathrm{m}}$ line. The map was cleaned down to $2 \sigma$ in a $2^{\prime \prime}$ radius circle centered at the source. The flux density measured inside an aperture of $r=1$ !" 3 from the usual map (clean components added on top of the residual) is $25 \%$ and $35 \%$ larger than the corrected one for the continuum and the line, respectively. The effect is more pronounced in sources with more extended emission and lower surface brightness, where a lot of flux remains in the residual. The numerical instability is evident in the continuum measurements at radii above $2^{\prime \prime}$, as both the dirty map and the residual map measurements approach the same value.

In Figure 8 we show the residual scaling corrected aperture fluxes for different continuum bands and atomic fine structure lines. Here we motivate the aperture radius with a diameter of 2 !" 6 (radius of 1 !" 3 ). The $[\mathrm{C} \mathrm{III}]_{158 \mu \mathrm{m}}$ emission is detected at the highest $\mathrm{S} / \mathrm{N}$ and is the most extended, the flux density reaches a plateau at $r=1$ !" 3 . The chosen aperture size recovers the flux density measured at the plateau within the error bars in all of the maps. We therefore opt for the same aperture in all measurements for the sake of consistent interpretation of probed spatial scales of line ratios, despite observations being at different resolutions.

\footnotetext{
11 http://www.astropy.org
} 

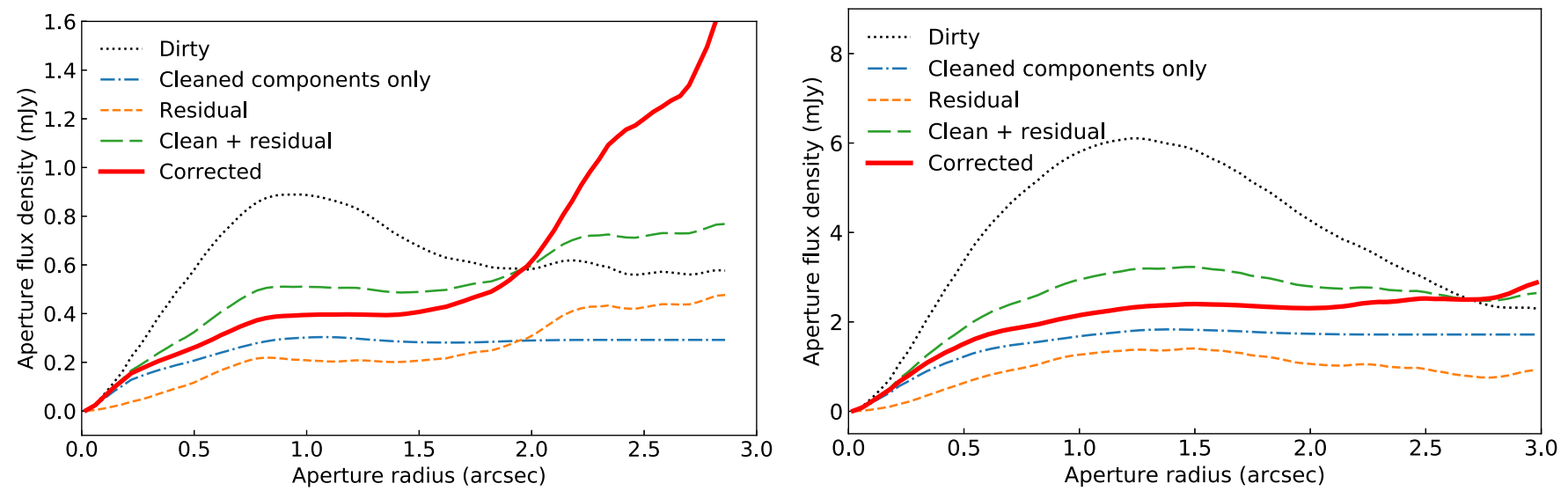

Figure 7. Aperture flux density of $\mathrm{J} 1342+0928$ observed with high resolution measured in various interferometric maps. Cleaning was performed down to $2 \sigma$ in a $2^{\prime \prime}$ radius circle. The clean beam size is used to convert from jansky per beam into jansky for all measurements. The green line represents flux density measured in the usual cleaned interferometric map. The red line demonstrates the effect of residual scaling correction. Left: continuum measurement at $231.5 \mathrm{GHz}$. Right: moment zero measurement of $[\mathrm{C} \mathrm{III}]_{158 \mu \mathrm{m}}$ integrated over $455 \mathrm{~km} \mathrm{~s}^{-1}$.
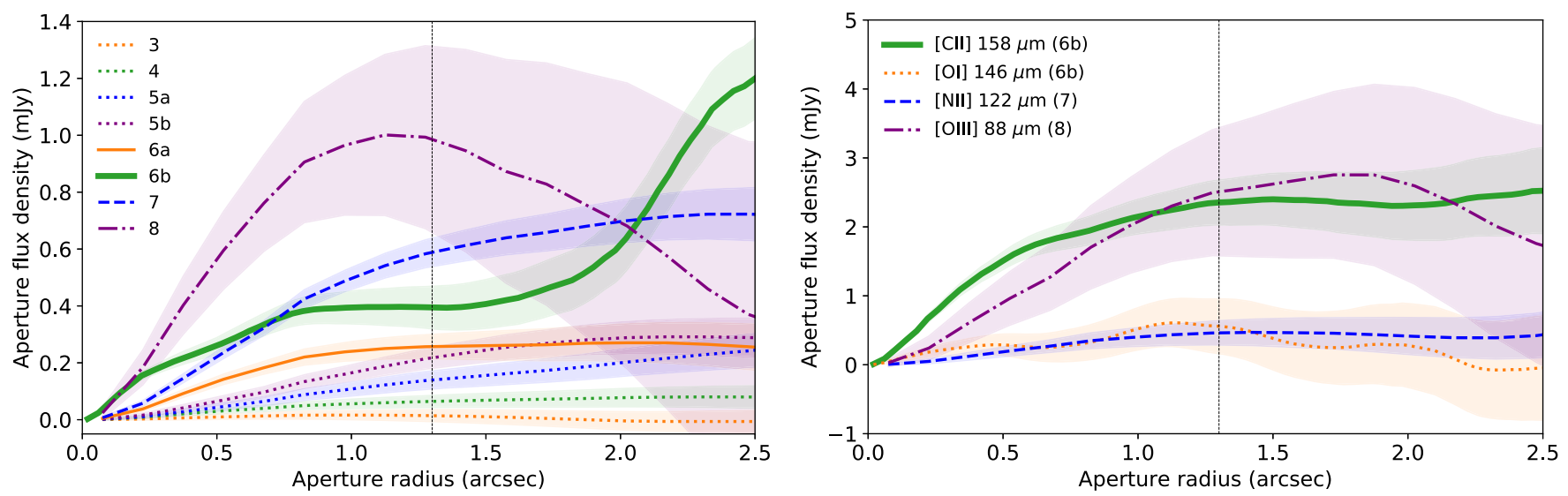

Figure 8. Flux density integrated inside an aperture as a function of its radius corrected with residual scaling. Left: continuum bands (see Table 1). Right: atomic fine structure lines. The vertical line shows the aperture used throughout the paper.

\section{Appendix B \\ Spectra and Nondetections of $\mathbf{J 1 3 4 2}+\mathbf{0 9 2 8}$}

In order to properly constrain the width of a spectral line, at least $6 \sigma$ is required in the central channel, so that the FWHM can be computed at a $3 \sigma$ significance. All of our lines, except $[\mathrm{C} \mathrm{III}]_{158 \mu \mathrm{m}}$, have less than $5 \sigma$ in the moment zero map integrated over $455 \mathrm{~km} \mathrm{~s}^{-1}$, and therefore have insufficient $\mathrm{S} / \mathrm{N}$ to fit a line profile in a meaningful way. However, since this work is a multiline spectral survey of an object, we show spectra of all considered lines in Figure 9 for completeness. The main goal of these spectra is to demonstrate the excess emission in individual channels. To maximize the $\mathrm{S} / \mathrm{N}$ they are extracted from a single pixel.

Also for completeness, we show integrated moment zero maps of our emission line nondetections in Figures 10 and 11. 

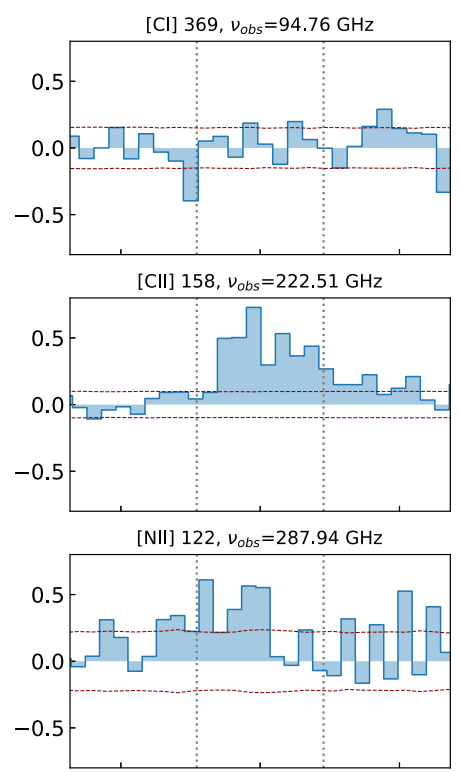

$\mathrm{CO}(7-6), v_{o b s}=94.44 \mathrm{GHz}$

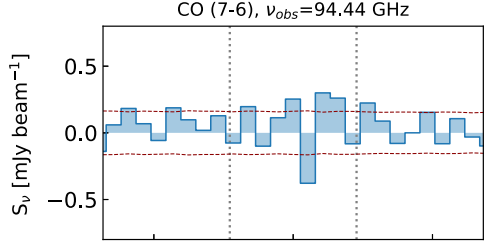

$\mathrm{CO}(10-9), v_{o b s}=134.87 \mathrm{GHz}$

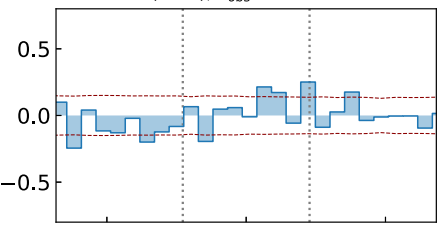

$\mathrm{CO}$ (14-13), $v_{o b s}=188.71 \mathrm{GHz}$

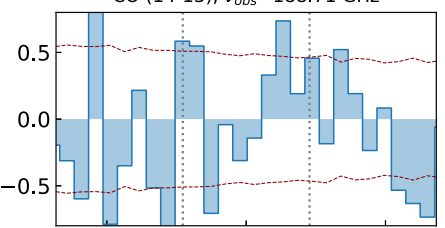

$\mathrm{CO}(16-15), v_{\text {obs }}=215.58 \mathrm{GHz}$

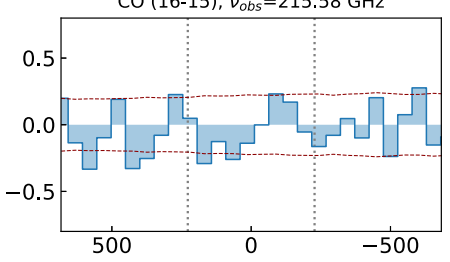

[NII] 205, $v_{\text {obs }}=171.07 \mathrm{GHz}$

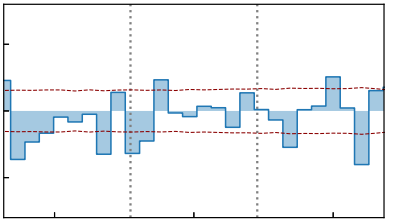

[OI] 146, $v_{\text {obs }}=241.19 \mathrm{GHz}$

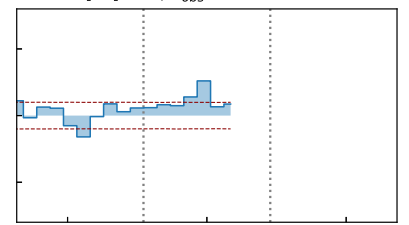

[OIII] 88, $v_{o b s}=397.25 \mathrm{GHz}$

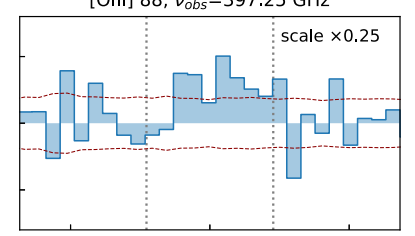

$\mathrm{CO}(8-7), v_{o b s}=107.92 \mathrm{GHz}$

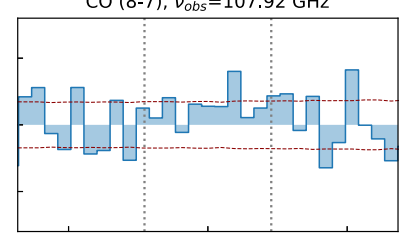

$\mathrm{CO}(11-10), v_{o b s}=148.34 \mathrm{GHz}$

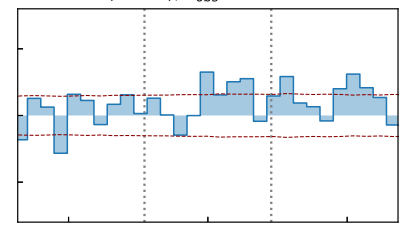

$\mathrm{CO}(15-14), v_{o b s}=202.15 \mathrm{GHz}$

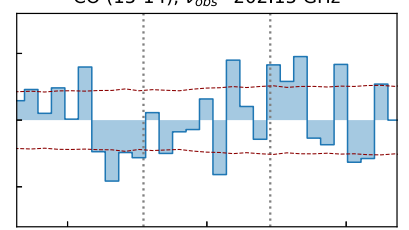

$\mathrm{CO}(17-16), v_{o b s}=229.01 \mathrm{GHz}$

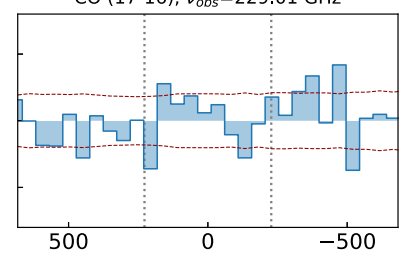

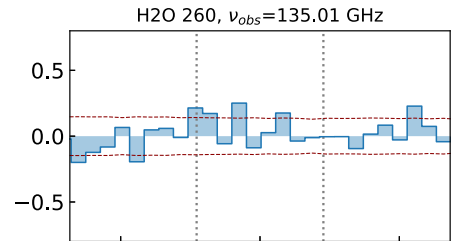

$\mathrm{H} 20175, v_{o b s}=201.00 \mathrm{GHz}$

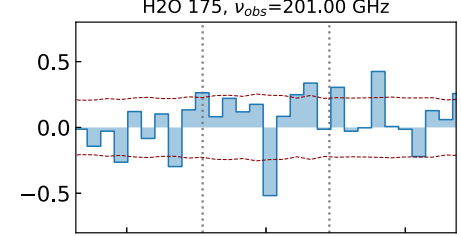

$\mathrm{OH} 163.4, v_{o b s}=214.81 \mathrm{GHz}$

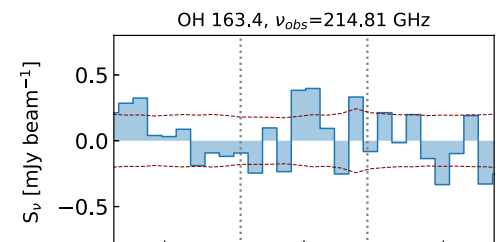

$\mathrm{OH}+330, v_{\text {obs }}=106.44 \mathrm{GHz}$

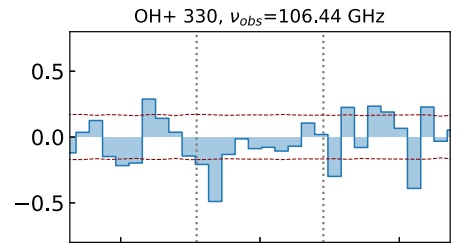

$\mathrm{OH}+153.0, v_{o b s}=229.42 \mathrm{GHz}$

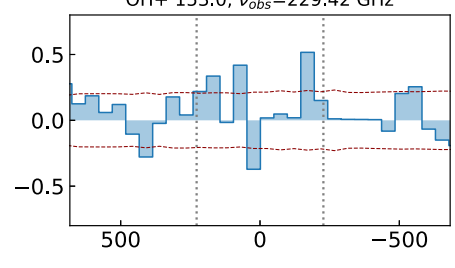

$\mathrm{H} 2 \mathrm{O} 258, v_{o b s}=136.15 \mathrm{GHz}$

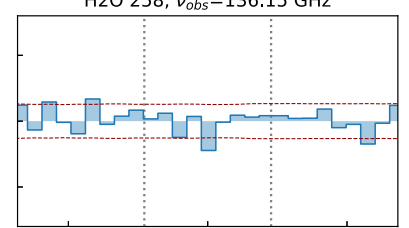

$\mathrm{H} 2 \mathrm{O} 156, v_{o b s}=224.72 \mathrm{GHz}$

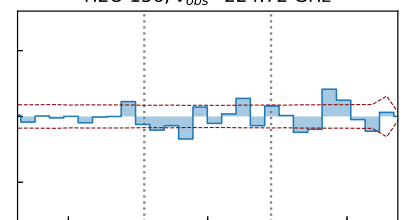

$\mathrm{OH} 163.1, v_{o b s}=215.17 \mathrm{GHz}$

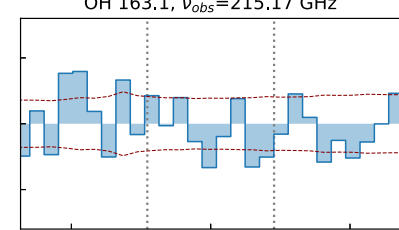

$\mathrm{OH}+153.5, v_{o b s}=228.72 \mathrm{GHz}$

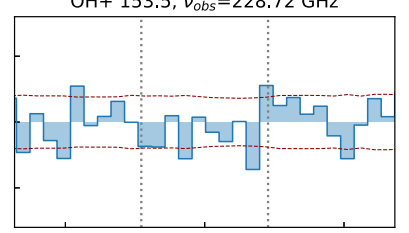

$\mathrm{OH}+152.4, v_{o b s}=230.36 \mathrm{GHz}$

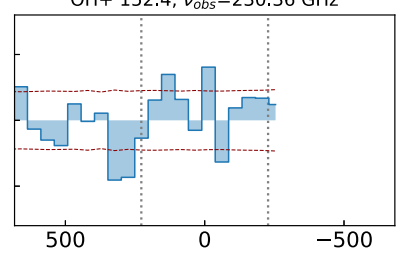

Figure 9. Single pixel spectra of atomic fine structure lines and $\mathrm{CO}$ lines (left two columns) and $\mathrm{H}_{2} \mathrm{O}, \mathrm{OH}$, and $\mathrm{OH}^{+}$(right two columns) of $\mathrm{J} 1342+0928$, extracted from the continuum subtracted cubes at the coordinate of the high resolution $231.5 \mathrm{GHz}$ continuum peak. The red lines show the rms measured inside each, approximately $50 \mathrm{~km} \mathrm{~s}^{-1}$ wide, channel. Vertical dashed lines encompass the $455 \mathrm{~km} \mathrm{~s}^{-1}$ width used to measure the line flux (see the text for details). Zero velocity corresponds to the redshifted line emission frequency $(z=7.5413)$. 

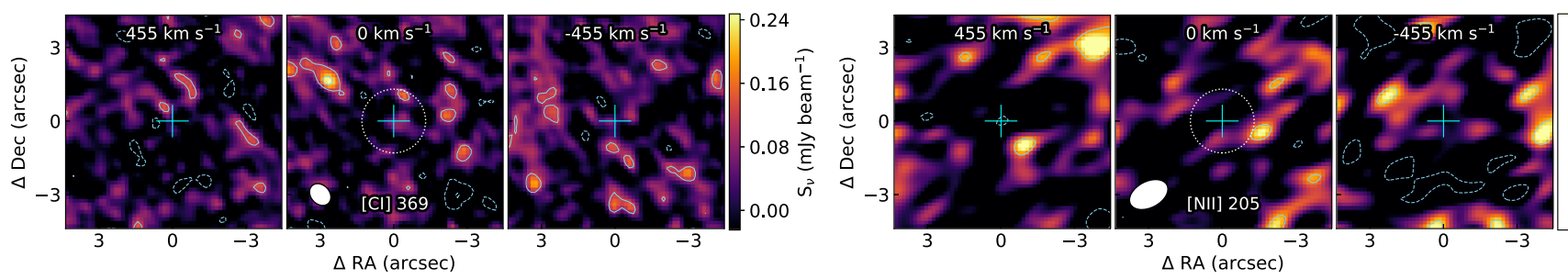

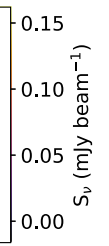
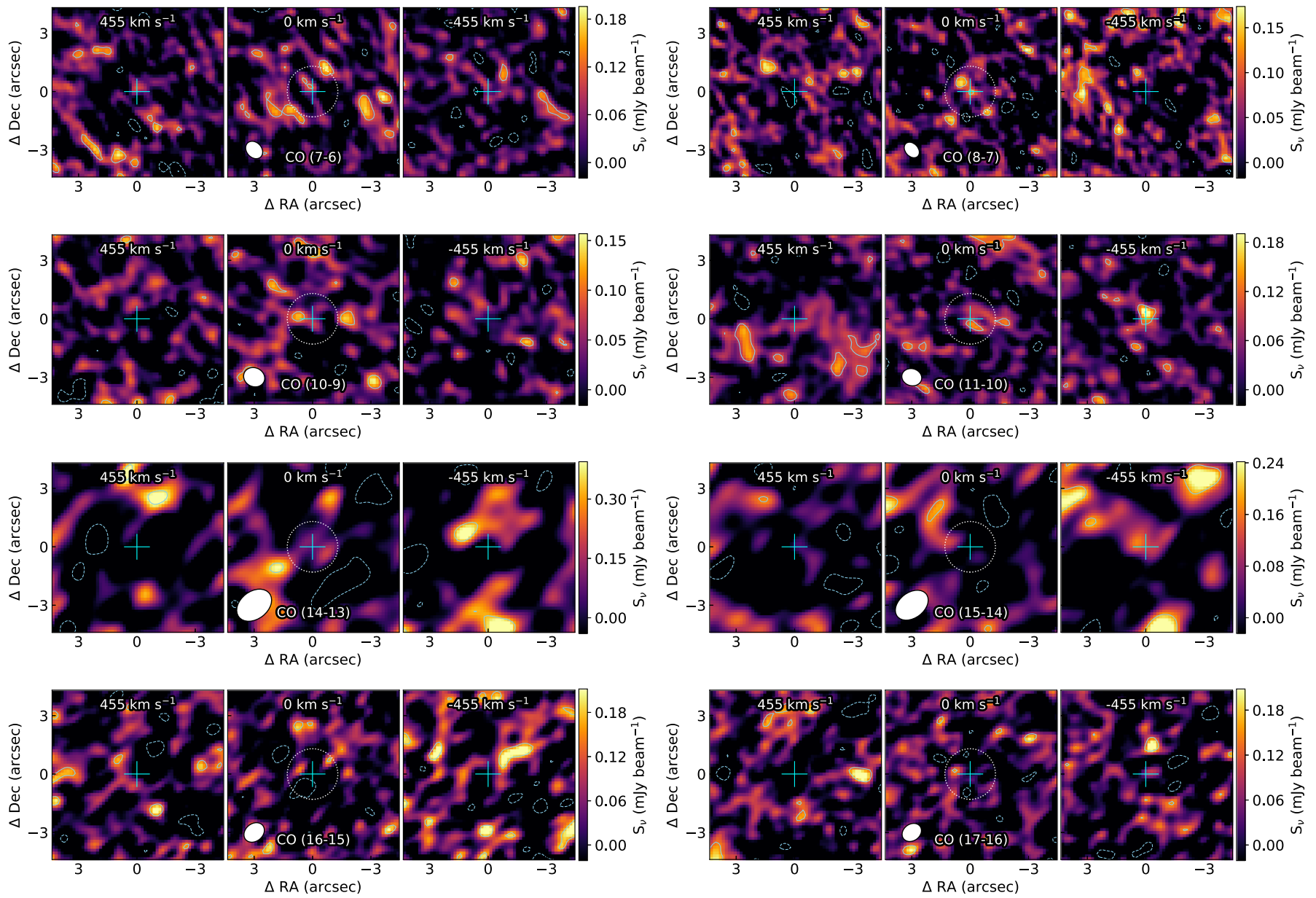

Figure 10. Nondetected atomic fine structure lines and CO lines in J1342+0928. Full (dashed) contours represent the $+(-) 2 \sigma, 4 \sigma$ emission significance. The cross marks the dust continuum emission center in the high-resolution data (same as in Figure 3). 

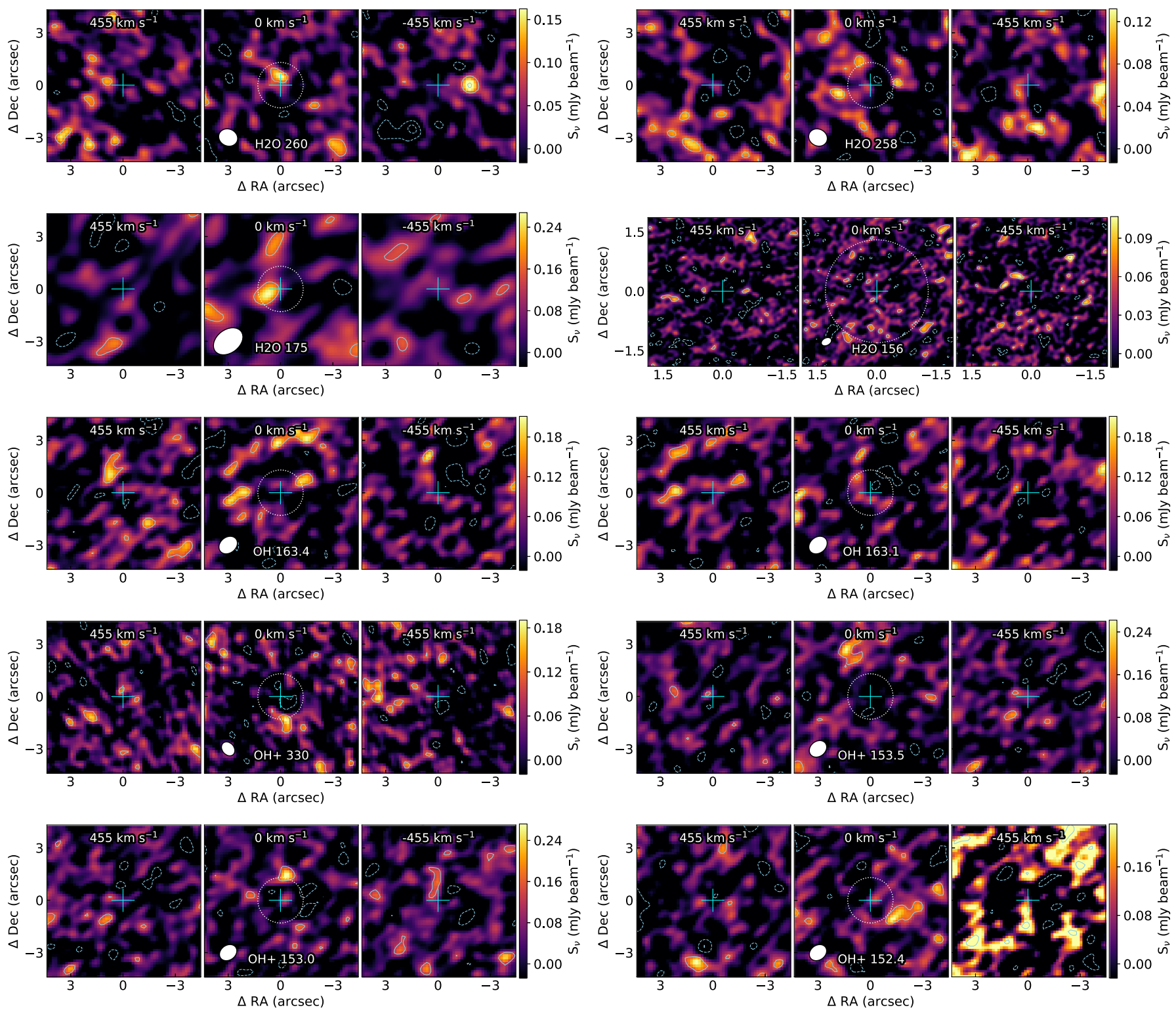

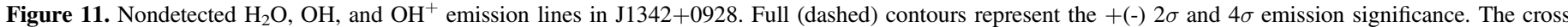
marks the dust continuum emission center in the high-resolution data (same as in Figure 3 ).

\section{ORCID iDs}

Mladen Novak (i) https://orcid.org/0000-0001-8695-825X Eduardo Bañados (1) https://orcid.org/0000-0002-2931-7824 Roberto Decarli (ii) https://orcid.org/0000-0002-2662-8803 Fabian Walter (1) https://orcid.org/0000-0003-4793-7880 Bram Venemans (i) https://orcid.org/0000-0001-9024-8322 Marcel Neeleman (i) https://orcid.org/0000-0002-9838-8191 Emanuele Paolo Farina (i) https://orcid.org/0000-0002-6822-2254 Chiara Mazzucchelli (i) https://orcid.org/0000-0002-5941-5214 Chris Carilli (i) https://orcid.org/0000-0001-6647-3861 Xiaohui Fan (1) https://orcid.org/0000-0003-3310-0131 Hans-Walter Rix (1) https://orcid.org/0000-0003-4996-9069 Feige Wang (1) https://orcid.org/0000-0002-7633-431X

\section{References}

Astropy Collaboration, Robitaille, T. P., Tollerud, E. J., et al. 2013, A\&A, 558, A33

Bañados, E., Novak, M., Neeleman, M., et al. 2019, ApJL, submitted
Bañados, E., Venemans, B. P., Decarli, R., et al. 2016, ApJS, 227, 11 Bañados, E., Venemans, B. P., Mazzucchelli, C., et al. 2018, Natur, 553, 473 Becker, G. D., Bolton, J. S., \& Lidz, A. 2015, PASA, 32, e045 Beelen, A., Cox, P., Benford, D. J., et al. 2006, ApJ, 642, 694 Bischetti, M., Piconcelli, E., Vietri, G., et al. 2017, A\&A, 598, A122 Bolatto, A. D., Wolfire, M., \& Leroy, A. K. 2013, ARA\&A, 51, 207 Brauher, J. R., Dale, D. A., \& Helou, G. 2008, ApJS, 178, 280 Carilli, C. L., \& Walter, F. 2013, ARA\&A, 51, 105

Carniani, S., Gallerani, S., Vallini, L., et al. 2019, arXiv:1902.01413 Carniani, S., Maiolino, R., Pallottini, A., et al. 2017, A\&A, 605, A42 Chabrier, G. 2003, PASP, 115, 763

da Cunha, E., Groves, B., Walter, F., et al. 2013, ApJ, 766, 13 Daddi, E., Dannerbauer, H., Liu, D., et al. 2015, A\&A, 577, A46

De Looze, I., Cormier, D., Lebouteiller, V., et al. 2014, A\&A, 568, A62 De Rosa, G., Decarli, R., Walter, F., et al. 2011, ApJ, 739, 56

De Rosa, G., Venemans, B. P., Decarli, R., et al. 2014, ApJ, 790, 145 Decarli, R., Walter, F., Venemans, B. P., et al. 2018, ApJ, 854, 97

Díaz-Santos, T., Armus, L., Charmandaris, V., et al. 2013, ApJ, 774, 68 Díaz-Santos, T., Armus, L., Charmandaris, V., et al. 2017, ApJ, 846, 32 Downes, D., \& Solomon, P. M. 1998, ApJ, 507, 615

Draine, B. T. 2011, ApJ, 732, 100

Dunne, L., Eales, S. A., \& Edmunds, M. G. 2003, MNRAS, 341, 589

Fan, X., Strauss, M. A., Becker, R. H., et al. 2006, AJ, 132, 117 
Ferkinhoff, C., Brisbin, D., Nikola, T., et al. 2011, ApJL, 740, L29

Ferkinhoff, C., Hailey-Dunsheath, S., Nikola, T., et al. 2010, ApJL, 714, L147

Ferland, G. J., Chatzikos, M., Guzmán, F., et al. 2017, RMxAA, 53, 385

Fischer, J., Sturm, E., González-Alfonso, E., et al. 2010, A\&A, 518, L41

Gallerani, S., Ferrara, A., Neri, R., \& Maiolino, R. 2014, MNRAS, 445, 2848

González-Alfonso, E., Fischer, J., Bruderer, S., et al. 2013, A\&A, 550, A25

Graciá-Carpio, J., Sturm, E., Hailey-Dunsheath, S., et al. 2011, ApJL, 728, L7

Hashimoto, T., Inoue, A. K., Mawatari, K., et al. 2019, PASJ, 70, psz049

Hashimoto, T., Inoue, A. K., Tamura, Y., et al. 2018, arXiv:1811.00030

Herrera-Camus, R., Bolatto, A., Smith, J. D., et al. 2016, ApJ, 826, 175

Herrera-Camus, R., Sturm, E., Graciá-Carpio, J., et al. 2018, ApJ, 861, 95

Hunter, J. D. 2007, CSE, 9, 90

Jiang, L., McGreer, I. D., Fan, X., et al. 2016, ApJ, 833, 222

Jorsater, S., \& van Moorsel, G. A. 1995, AJ, 110, 2037

Katz, H., Galligan, T. P., Kimm, T., et al. 2019, MNRAS, 487, 5902

Katz, H., Kimm, T., Sijacki, D., \& Haehnelt, M. G. 2017, MNRAS, 468, 4831

Kennicutt, R. C., \& Evans, N. J. 2012, ARA\&A, 50, 531

Kennicutt, R. C. J. 1998, ARA\&A, 36, 189

Kurk, J. D., Walter, F., Fan, X., et al. 2007, ApJ, 669, 32

Liu, L., Weiß, A., Perez-Beaupuits, J. P., et al. 2017, ApJ, 846, 5

Malhotra, S., Helou, G., Stacey, G., et al. 1997, ApJL, 491, L27

Maloney, P. R., Hollenbach, D. J., \& Tielens, A. G. G. M. 1996, ApJ, 466, 561

Marrone, D. P., Spilker, J. S., Hayward, C. C., et al. 2018, Natur, 553, 51

Matsuoka, Y., Strauss, M. A., Kashikawa, N., et al. 2018, ApJ, 869, 150

Mazzucchelli, C., Bañados, E., Venemans, B. P., et al. 2017, ApJ, 849, 91

McMullin, J. P., Waters, B., Schiebel, D., Young, W., \& Golap, K. 2007, adass

XVI, 376 ed. R. A. Shaw, F. Hill, \& D. J. Bell, 127

Meijerink, R., \& Spaans, M. 2005, A\&A, 436, 397

Meijerink, R., Spaans, M., \& Israel, F. P. 2007, A\&A, 461, 793

Oberst, T. E., Parshley, S. C., Stacey, G. J., et al. 2006, ApJL, 652, L125

Pereira-Santaella, M., Rigopoulou, D., Farrah, D., Lebouteiller, V., \& Li, J. 2017, MNRAS, 470, 1218

Price-Whelan, A. M., Sipőcz, B. M., Günther, H. M., et al. 2018, AJ, 156, 123

Rémy-Ruyer, A., Madden, S. C., Galliano, F., et al. 2014, A\&A, 563, A31
Riechers, D. A., Bradford, C. M., Clements, D. L., et al. 2013, Natur, 496, 329 Riechers, D. A., Walter, F., Bertoldi, F., et al. 2009, ApJ, 703, 1338

Rigopoulou, D., Pereira-Santaella, M., Magdis, G. E., et al. 2018, MNRAS, 473, 20

Rosenberg, M. J. F., van der Werf, P. P., Aalto, S., et al. 2015, ApJ, 801, 72

Rybak, M., Calistro Rivera, G., Hodge, J. A., et al. 2019, ApJ, 876, 112

Sandstrom, K. M., Leroy, A. K., Walter, F., et al. 2013, ApJ, 777, 5

Savage, B. D., \& Sembach, K. R. 1996, ARA\&A, 34, 279

Sijacki, D., Vogelsberger, M., Genel, S., et al. 2015, MNRAS, 452, 575

Solomon, P. M., Downes, D., Radford, S. J. E., \& Barrett, J. W. 1997, ApJ, 478,144

Stefan, I. I., Carilli, C. L., Wagg, J., et al. 2015, MNRAS, 451, 1713

Tadaki, K.-I., Iono, D., Hatsukade, B., et al. 2019, ApJ, 876, 1

Tielens, A. G. G. M., \& Hollenbach, D. 1985, ApJ, 291, 722

van der Vlugt, D., \& Costa, T. 2019, arXiv:1903.04544

van der Werf, P. P., Berciano Alba, A., Spaans, M., et al. 2011, ApJL, 741, L38

Venemans, B. P., Walter, F., Decarli, R., et al. 2017a, ApJ, 845, 154

Venemans, B. P., Walter, F., Decarli, R., et al. 2017b, ApJL, 851, L8

Venemans, B. P., Walter, F., Decarli, R., et al. 2017c, ApJ, 837, 146

Volonteri, M. 2012, Sci, 337, 544

Walter, F., \& Brinks, E. 1999, AJ, 118, 273

Walter, F., Brinks, E., de Blok, W. J. G., et al. 2008, AJ, 136, 2563

Walter, F., Riechers, D., Novak, M., et al. 2018, ApJL, 869, L22

Walter, F., Weiß, A., Downes, D., Decarli, R., \& Henkel, C. 2011, ApJ, 730,18

Wang, R., Wagg, J., Carilli, C. L., et al. 2013, ApJ, 773, 44

Wang, R., Wu, X.-B., Neri, R., et al. 2016, ApJ, 830, 53

Weiß, A., Downes, D., Henkel, C., \& Walter, F. 2005, A\&A, 429, L25

Weiß, A., Downes, D., Neri, R., et al. 2007, A\&A, 467, 955

Weiß, A., Henkel, C., Downes, D., \& Walter, F. 2003, A\&A, 409, L41

Willott, C. J., Bergeron, J., \& Omont, A. 2015, ApJ, 801, 123

Yang, J., Venemans, B., Wang, F., et al. 2019, arXiv:1907.00385

Zanella, A., Daddi, E., Magdis, G., et al. 2018, MNRAS, 481, 1976 